\title{
On Benchmarking of Simulations of Particle Transport in ITER
}

Yong-Su Na ${ }^{1}$, F. Koechl ${ }^{2}$, A.R. Polevoi ${ }^{3}$, C.S. Byun ${ }^{1}$, D. H. Na ${ }^{1}$, J. Seo ${ }^{1}$, F. Felici ${ }^{4}$, A. Fukuyama ${ }^{5}$, J. Garcia ${ }^{6}$, N. Hayashi ${ }^{7}$, C.E. Kessel ${ }^{8}$, T. Luce ${ }^{3}$, J.M. Park ${ }^{9}$, F. Poli ${ }^{8}$, O. Sauter ${ }^{10}$, A.C.C. Sips ${ }^{11}$, P. Strand $^{12}$, A. Teplukhina ${ }^{10}$, I. Voitsekhovitch ${ }^{13}$, A. Wisitsorasak ${ }^{14}$, X. Yuan ${ }^{8}$, and the ITPA Topical Group on Integrated Operation Scenarios

${ }^{1}$ Department of Nuclear Engineering, Seoul National University, Seoul, Korea

${ }^{2}$ United Kingdom Atomic Energy Authority, Culham Centre for Fusion Energy, Culham Science Centre, Abingdon, Oxon, OX14 3DB, UK

${ }^{3}$ ITER Organisation, Route de Vinon-sur-Verdon, CS 90 046, 13067 St. Paul Lez Durance Cedex, France

${ }^{4}$ Eindhoven University of Technology, Eindhoven, Netherlands

${ }^{5}$ Kyoto University, Kyoto, Japan

${ }^{6}$ CEA, IRFM, 13108 Saint-Paul-lez-Durance, France

${ }^{7}$ National Institutes for Quantum and Radiological Science and Technology, Naka, Ibaraki, 311-0193, Japan

${ }^{8}$ Princeton Plasma Physics Laboratory, Princeton, New Jersey, USA

${ }^{9}$ Oak Ridge National Laboratory, Oak Ridge, Tennessee, USA

${ }^{10}$ Ecole Polytechnique Fédérale de Lausanne (EPFL), Swiss Plasma Center (SPC), CH-1015

Lausanne, Switzerland

${ }^{11}$ JET Exploitation Unit, Culham Science Centre, Abingdon OX14 3DB, UK

${ }^{12}$ Department of Space, Earth and Environment, Chalmers University of Technology, Sweden

${ }^{13}$ EUROfusion, Garching, Germany

${ }^{14}$ King Mongkut's University of Technology Thonburi, Bangkok, Thailand

E-mail contact of main author: ysna@snu.ac.kr

\begin{abstract}
We report results of benchmarking of core particle transport simulations by a collection of codes widely used in transport modelling of tokamak plasmas. Our analysis includes formulation of transport equations, difference between electron and ion solvers, comparison of modules of the
\end{abstract}


pellet and edge gas fuelling on the ITER baseline scenario. During the first phase of benchmarking we address the particle transport effects in the stationary phase. Firstly, simulations are performed with identical sources, sinks, transport coefficients, and boundary conditions prescribed in the flattop H-mode phase. The transformation of ion particle transport equations is introduced so to directly compare their results to electron transport solvers. Secondly, the pellet fuelling models are benchmarked in various conditions to evaluate the dependency of the pellet deposition on the pellet volume, injection side, pedestal and separatrix parameters. Thirdly, edge gas fuelling is benchmarked to assess sensitivities of source profile predictions to uncertainties in plasma conditions and detailed model assumptions. At the second phase, we address particle transport effects in the time-evolving plasma including the current ramp-up to the ramp-down phase. The ion and the electron solvers are benchmarked together. Differences between the simulation results of the solvers are investigated in terms of equilibrium, grid resolution, radial coordinate, radial grid distribution, and plasma volume evolution term. We found that the selection of the radial coordinate can yield prominent differences between the solvers mainly due to differences in the edge grid distribution. The simulations reveal that electron and ion solvers predict noticeably different density peaking for the same diffusion and pinch velocity while with the peaked profile of helium, expected in fusion reactors. The fuelling benchmarking shows that gas puffing is not efficient for core fuelling in H-modes and density control should be done by the high field side pellet injection in contrast to present machines.

\section{Introduction}

The time evolution of the D-T fuel profile in the plasma core has a strong impact on the fusion plasma performance in a fusion reactor. There are several important issues in particle transport on ITER that need to be addressed [1]. The complexity of particle transport including diffusion and convection in different parts of the discharge poses a question as to how the density is established in L-mode, how it affects the L-H transition, how it is controlled in the current flattop H-mode ultimately, and how it evolves during the current ramp-down including the H-L transition [2-5]. Particularly, the density profile evolution at the L-H transition has significant implications for entering and staying in H-mode depending on the ratio of the power flux to the scrape-off-layer (SOL) to the L-H threshold, $\mathrm{P}_{\text {sol }} / \mathrm{P}_{\mathrm{LH}}$ [6]. Developing credible burn control strategies for the $\mathrm{H}$ - 
mode in the current flattop phase depends sensitively on the particle balance of the mixed D-T fuels, He and impurities. In ITER, the neutral beam injection (NBI) does not play a noticeable role neither in the global particle balance [7], nor for the central fuelling. Moreover, the SOLPS modelling [7] predicts dramatic reduction of the gas penetrated from the edge, making the pellet injection the main tool for the density control in the H-mode plasmas, though the gas penetrated from the edge still can play the dominant role for the L-mode operation. Features like the recycling and penetration of $\mathrm{He}$ and the fuel into the core plasma are central to understanding the dilution and tritium burnup. The SOL/divertor plasma and its interactions with plasma facing components will set the boundary conditions for the core transport. Eventually, the particle transport alters the heat and the momentum transport so all these non-linear connections need to be understood simultaneously to predict the fusion plasma performance precisely.

To address these issues, 1.5-D particle transport modelling is essential with integrated transport codes. Although progress has been made in predictive particle transport modelling of core plasmas $[8,9]$, and in the area of 2-D SOL/divertor modelling, it is still a much less mature area compared to heat transport. Particle transport in the core plasma is often not treated despite its importance in integrated scenario simulations due to 1) uncertainties of measurements to determine the separatrix density and the 3-D fuel sources to validate transport models, 2) difficulties on properly determining the diffusive and pinch parts of the flux, 3) complexity of multi-species impurity transport, and 4) complicated interaction with the SOL, divertor, and plasma facing materials. Optimisation of the fuelling scenario for ITER requires sufficiently accurate numerical solvers with appropriate description of particle sources, sinks, boundary conditions, and integration in the codes for simulations of self-consistent plasma evolutions. In this context, the international tokamak physics activity (ITPA) integrated operation scenario (IOS) topical group is pursuing particle transport as an important component of integrated modelling, as part of a broader scheme to expand toward impurity, alpha particle, and momentum transport.

The particle transport code benchmarking is carried out with various integrated modelling codes used for the ITER scenario simulations as was done for heat transport [10]. The purpose of the benchmark is to identify the differences in treatment of particle transport between codes in conditions close to those expected in ITER, and to compare the sensitivity of particle transport predictions to modelling assumptions and to reveal the relevant critical issues to be clarified in 
dedicated modelling and experiments on present machines so to predict particle transport more accurately in tokamak fusion plasmas.

The particle transport codes in the integrated modelling tools comprise solvers for ion or electron particle transport with consistent metric and transport coefficients, modules for particle sources from the pellet fuelling and the edge gas puffing, modules for particle sinks with edge localised mode (ELM), interfaces with SOL/divertor transport codes or modules for predicting the H-mode pedestal structure or for simulating the edge boundary condition consistent with the heat and particle out-fluxes or modules. The benchmarking includes comparison of the pellet and the gas fuelling modules as well as the particle transport solvers to discuss possible impact of the plasma parameters on the simulations of the pellet deposition and transport of the neutral gas due to the charge exchange and recombination. The benchmark will enable the assessment of the impact of the variety of model assumptions used in different codes on the divergence of plasma performance prediction in ITER-like conditions. These systematic multi-code studies of the impact of all aspects of the particle transport treatment were never done before.

At the first phase of benchmarking described here, we address the particle transport effects related to the stationary current flattop phase of the H-mode operation for plasma parameters expected in the baseline ITER scenario with the vacuum magnetic field $B_{0}=5.3 \mathrm{~T}$ at major radius $R_{0}=6.2 \mathrm{~m}$ and plasma current $I_{\mathrm{p}}=15 \mathrm{MA}$. The particle transport solvers are benchmarked in a stationary target plasma at a fixed equilibrium with prescribed particle transport coefficients, sources, and boundary conditions. The study begins with unification of definitions between particle transport solvers by identifying similarities and differences between them. For the benchmark, the particle flow balance is checked firstly in each particle transport solver in a stationary condition. Secondly, the ion transport solvers, which solve the ion particle transport equation, are benchmarked with each other, then with the electron transport solvers, which solve the electron particle transport equation, by modifying the pinch term in the ion transport equation to replace the ion flux by the electron one for allowing direct comparisons with the electron solvers. Thirdly, the pellet fuelling models are benchmarked in various conditions to evaluate the sensitivity of the pellet deposition profile on the pellet volume, the injection side (high field side (HFS) or low field side (LFS)), the pedestal parameters, and the separatrix parameters. Fourthly, the model for the fuelling by $\mathrm{D}$ and $\mathrm{T}$ neutrals penetrated to the core through the separatix are benchmarked together with the 
sensitivity studies for each of the models on the pedestal and edge plasma parameters for the range expected in the ITER in the L-and H-mode DT operation.

At the second phase of benchmarking, we address the particle transport in the time-evolving scenario covering from the L-mode ramp-up phase, L-H transition, flattop H-mode phase, H-L transition, and L-mode ramp-down phase. The ion and the electron solvers are benchmarked together with prescribed evolution of the plasma configuration, particle transport coefficients, sources, and boundary conditions.

The differences observed between codes during the benchmarking are discussed. Firstly, the effect of the equilibrium and grid resolution is evaluated. Secondly, the solvers with $\rho$ and normalised $\rho, \rho_{\mathrm{N}}=\left(\Phi / \Phi_{\mathrm{a}}\right)^{1 / 2}$ are compared and the effect of the grid distribution is investigated in the time-evolving case, where $\rho=\left(\Phi / \pi \mathrm{B}_{0}\right)^{1 / 2}$ and $\Phi, \Phi_{\mathrm{a}}$, and $\mathrm{B}_{0}$ are the toroidal magnetic flux, $\Phi$ at the plasma boundary, and the vacuum magnetic field strength at the geometric centre of the vacuum chamber, respectively. Lastly, the role of the term regarding the time-evolving magnetic configuration, the so-called volume evolution term, is studied.

The paper is organised as follows. In section 2, we compare the equations used in particle transport solvers and describe the setup of the benchmarking tasks for the codes. The benchmark results of simulations in the stationary phase for the ion and electron solvers, as well as the sensitivity studies of pellet and gas fuelling predictions are presented for prescribed target plasma parameters in section 3. The results of time-evolving plasmas are also compared in section 3 . The possible reasons for the discrepancies between particle transport solvers observed during the benchmark are discussed in terms of the equilibrium, grid, radial coordinate, and volume evolution term in section 4 . The impact of the integrated plasma performance on the particle transport is also discussed in section 4. Finally, the conclusions are drawn in section 5.

\section{Setup for particle transport benchmark study}

\subsection{Unification of definitions}

The particle transport is modelled by the 1-D transport equations for plasma species with diffusivities, pinch velocities, boundary conditions, and particle sources and sinks. The particle 
transport equations are compared as below for unification of definitions between transport solvers involved in this benchmarking study (see table 1), where the differences are highlighted in bold red in equations (1)-(7). Note that in the case of the "ion solvers" the density transport is modelled for the ions and the electron density is calculated from the quasi-neutrality condition, $n_{e}=\Sigma n_{k} Z_{k}$, where $\sum n_{k} Z_{k}$ is the sum of the all ion species with the charge sate $Z_{k}$, for the "electron solver" vice versa. A further simplification which is often used in the particle transport simulations is the prescription of the impurity densities as a fraction of the electron density, $n_{k}=f_{k} n_{e}$, and consideration of the fuel ions as a single species. Such simplification will be discussed below to illustrate the difference of the electron and ion solver predictions for the case of the fusion reactor.

$\frac{1}{V^{\prime}}\left(\frac{\partial}{\partial t}-\frac{1}{2 \Phi_{a}} \frac{d \Phi_{a}}{d t} \frac{\partial}{\partial \rho_{N}} \rho_{N}\right)\left(V^{\prime} n_{k}\right)+\frac{1}{V^{\prime}} \frac{\partial}{\partial \rho_{N}}\left[V^{\prime}\left\langle\left(\nabla \rho_{N}\right)^{2}\right\rangle\left(-D_{k} \frac{\partial n_{k}}{\partial \rho_{N}}+n_{k} v_{k}\right)\right]=S_{k}$

implemented in ASTRA v.7.0 [12], ETS [13] $(\mathrm{k}=\mathrm{e}, \mathrm{i})$, and TRANSP/PTSOLVER v.18.1 [14] (k $=\mathrm{e})$, where $\mathrm{k}=\mathrm{e}$ and $\mathrm{i}$ correspond to the electron and the ion particle transport solver, respectively.

$\frac{1}{V^{\prime}}\left(\frac{\partial}{\partial t}-\frac{1}{2 \Phi_{a}} \frac{d \Phi_{a}}{d t} \frac{\partial}{\partial \rho_{N}} \rho_{N}\right)\left(V^{\prime} n_{k}\right)+\frac{1}{V^{\prime}} \frac{\partial}{\partial \rho_{N}}\left[V^{\prime}\left(-D_{k} \frac{\partial n_{k}}{\partial \rho_{N}}\left\langle\left(\nabla \rho_{N}\right)^{2}\right\rangle+n_{k} v_{k}\left\langle\left|\nabla \rho_{N}\right|\right\rangle\right)\right]=S_{k}$

implemented in RAPTOR particle transport solver $(\mathrm{k}=\mathrm{e}, \mathrm{i})$ [15],

$\frac{1}{V^{\prime}}\left(\frac{\partial}{\partial t}-\frac{1}{2 \Phi_{a}} \frac{d \Phi_{a}}{d t} \frac{\partial}{\partial \rho_{N}} \rho_{N}\right)\left(V^{\prime} n_{e}\right)+\frac{1}{V^{\prime}} \frac{\partial}{\partial \rho_{N}}\left[V^{\prime}\left(-D_{i} \frac{\partial n_{i}}{\partial \rho_{N}}\left\langle\left(\nabla \rho_{N}\right)^{2}\right\rangle-n_{i} v_{i}\left\langle\left|\nabla \rho_{N}\right|\right\rangle\right)\right]=S_{i}-S_{D T}$

implemented in TOPICS ion particle transport solver [16],

$\frac{1}{V^{\prime}} \frac{\partial}{\partial t}\left(V^{\prime} n_{e}\right)+\frac{1}{V^{\prime}} \frac{\partial}{\partial \rho}\left[V^{\prime}\left\langle(\nabla \rho)^{2}\right\rangle\left(-D_{e} \frac{\partial n_{e}}{\partial \rho}-n_{e} v_{e}\right)\right]=S_{e}$

implemented in CRONOS electron particle transport solver [17],

$\frac{1}{V^{\prime}} \frac{\partial}{\partial t}\left(V^{\prime} n_{e}\right)+\frac{1}{V^{\prime}} \frac{\partial}{\partial \rho}\left[V^{\prime}\left\langle(\nabla \rho)^{2}\right\rangle\left(-D_{e} \frac{\partial n_{e}}{\partial \rho}+n_{e} v_{e}\right)\right]=S_{e}$ 
implemented in FASTRAN electron particle transport solver [18],

$\frac{1}{V^{\prime}} \frac{\partial}{\partial t}\left(V^{\prime} n_{i}\right)+\frac{1}{V^{\prime}} \frac{\partial}{\partial \rho}\left[V^{\prime}\left(-D_{i} \frac{\partial n_{i}}{\partial \rho}\left\langle(\nabla \rho)^{2}\right\rangle+n_{i} v_{i}\langle|\nabla \rho|\rangle\right)\right]=S_{i}-S_{D T}$

implemented in JINTRAC ion transport solver [19],

$\frac{1}{V^{\prime}} \frac{\partial}{\partial t}\left(V^{\prime} n_{e}\right)+\frac{1}{V^{\prime}} \frac{\partial}{\partial \rho}\left[V^{\prime}\left(-D_{e} \frac{\partial n_{e}}{\partial \rho}\left\langle(\nabla \rho)^{2}\right\rangle+n_{e} v_{e}\langle|\nabla \rho|\rangle\right)\right]=S_{e}$

$\frac{1}{V^{\prime}} \frac{\partial}{\partial t}\left(V^{\prime} n_{i}\right)+\frac{1}{V^{\prime}} \frac{\partial}{\partial \rho}\left[V^{\prime}\left(-D_{i} \frac{\partial n_{i}}{\partial \rho}\left\langle(\nabla \rho)^{2}\right\rangle+n_{i} v_{i}\langle|\nabla \rho|\rangle\right)\right]=S_{i}-S_{D T}$

implemented in TASK/TR electron or ion particle transport solver [20],

Here $n_{e}$ and $n_{i}$ are the electron and the fuel ion density, respectively. The fuel ion density, $n_{i}=n_{D}$ $+\mathrm{n}_{\mathrm{T}}$ and $\mathrm{n}_{\mathrm{D}}=\mathrm{n}_{\mathrm{T}}$ are assumed, where $\mathrm{n}_{\mathrm{D}}$ and $\mathrm{n}_{\mathrm{T}}$ are the deuteron and the triton density, respectively. $\mathrm{V}$ is the plasma volume, $\mathrm{D}_{\mathrm{k}}$ and $\mathrm{vk}_{\mathrm{k}}$, and $\mathrm{S}_{\mathrm{k}}$ are the diffusivity, the pinch velocity, and the sum of particle sources and sinks including ionisation, recombination of $\mathrm{k}$ species except for the fuel ion density loss due to DT fusion reactions, SDT, respectively. Note that for sources and sinks the quasineutrality condition takes the form, $\Sigma S_{k} Z_{k}=S_{e}$. The boundary conditions are given at the centre as $(\partial n / \partial \rho)=0$. At the edge the boundary conditions can be of the first kind with prescribed values of densities, $n_{k a}(t)$ with quasi-neutrality, $\Sigma n_{k a}(t) Z_{k}=n_{e a}(t)$, or of the third kind, with prescribed boundary fluxes, $\Gamma_{k a}(t)$ with quasi-neutrality, $\Sigma \Gamma_{k a}(t) Z_{k}=\Gamma_{e a}(t)$ (definition of the fluxes shown in equations (8)-(11)). As shown above, the differences between solvers are identified as 1) type of particles, 2) radial coordinate, 3) description of the plasma shape and volume evolution, 4) sign of the pinch velocity, 5) metric coefficients, $\left\langle(\nabla \rho)^{2}\right\rangle$ and $\langle|\nabla \rho|\rangle$ used for diffusive and convective terms, 6) fuel ion density loss term due to fusion reactions. Note that solvers (1), (4), (5) will predict the same density decay length $L_{n}=-R n^{\prime} / n=R v / D$ for the same transport coefficients in stationary conditions $\left(d \Phi_{a} / d t=0\right)$, considering different sign conventions for v. Likewise, the same $L_{n}$ will be obtained with solvers (2), (3), (6), (7) for $\mathrm{k}=\mathrm{i}$, neglecting SDT. To deal with the most outstanding difference of the type of solvers, we benchmark the ion transport solvers first. Then to benchmark the ion and the electron solvers for the stationary solutions we replace the ion fluxes by electron 
fluxes introducing necessary terms in the convective part of the ion transport solvers to emulate the electron transport solver in the frame of the ion solvers. The modified ion pinch velocities are formulated in terms of the electron pinch velocity and diffusivity for corresponding definitions of the ion particle flux, $\Gamma_{i}$ as below.

$$
\begin{aligned}
& \mathrm{v}_{\mathrm{i}}=-\mathrm{v}_{\mathrm{e}} \frac{n_{e}}{n_{i}}-\frac{D_{e}}{n_{i}}\left(\frac{\partial n_{e}}{\partial \rho}-\frac{\partial n_{i}}{\partial \rho}\right) \text { for } \Gamma_{i} / V^{\prime}=-D_{i} \frac{\partial n_{i}}{\partial \rho}\left\langle(\nabla \rho)^{2}\right\rangle+n_{i} v_{i}\left\langle(\nabla \rho)^{2}\right\rangle \\
& \mathrm{v}_{\mathrm{i}}=\mathrm{v}_{\mathrm{e}} \frac{n_{e}}{n_{i}}+\frac{D_{e}}{n_{i}}\left(\frac{\partial n_{e}}{\partial \rho}-\frac{\partial n_{i}}{\partial \rho}\right) \text { for } \Gamma_{i} / V^{\prime}=-D_{i} \frac{\partial n_{i}}{\partial \rho}\left\langle(\nabla \rho)^{2}\right\rangle-n_{i} v_{i}\left\langle(\nabla \rho)^{2}\right\rangle \\
& \mathrm{v}_{\mathrm{i}}=-\mathrm{v}_{\mathrm{e}} \frac{n_{e}}{n_{i}} \frac{\left\langle(\nabla \rho)^{2}\right\rangle}{\langle|\nabla \rho|\rangle}-\frac{D_{e}}{n_{i}}\left(\frac{\partial n_{e}}{\partial \rho}-\frac{\partial n_{i}}{\partial \rho}\right) \frac{\left\langle(\nabla \rho)^{2}\right\rangle}{\langle|\nabla \rho|\rangle} \text { for } \Gamma_{i} / V^{\prime}=-D_{i} \frac{\partial n_{i}}{\partial \rho}\left\langle(\nabla \rho)^{2}\right\rangle+n_{i} v_{i}\langle|\nabla \rho|\rangle \\
& \mathrm{v}_{\mathrm{i}}=\mathrm{v}_{\mathrm{e}} \frac{n_{e}}{n_{i}} \frac{\left\langle(\nabla \rho)^{2}\right\rangle}{\langle|\nabla \rho|\rangle}+\frac{D_{e}}{n_{i}}\left(\frac{\partial n_{e}}{\partial \rho}-\frac{\partial n_{i}}{\partial \rho}\right) \frac{\left\langle(\nabla \rho)^{2}\right\rangle}{\langle|\nabla \rho|\rangle} \text { for } \Gamma_{i} / V^{\prime}=-D_{i} \frac{\partial n_{i}}{\partial \rho}\left\langle(\nabla \rho)^{2}\right\rangle-n_{i} v_{i}\langle|\nabla \rho|\rangle
\end{aligned}
$$

In this way, the electron flux that would be obtained with an electron transport equation with fixed impurities can be calculated with the ion transport solvers. Note that the edge boundary condition in the ion solvers, $\mathrm{n}_{\mathrm{ia}}$ is properly adjusted to satisfy the prescribed boundary condition of the electron solvers, $\mathrm{n}_{\text {ea }}$ through quasi-neutrality.

The differences from the sign of the pinch term and the toroidal metric could be handled in each code so to ensure the same setup in simulations for the benchmarking study. The fuel density loss term due to fusion reactions which can affect the results significantly depending on the fusion reaction rate is switched off for consistency.

Table 1. The list of the benchmarked codes with available type of solvers

\begin{tabular}{|c|c|c|}
\hline Code & Solver & Reference \\
\hline ASTRA v.6 & $\mathrm{n}_{\mathrm{e}}$ or $\mathrm{n}_{\mathrm{i}}$ & {$[11]$} \\
\hline ASTRA v.7 & $\mathrm{n}_{\mathrm{e}}$ or $\mathrm{n}_{\mathrm{i}}$ & {$[12]$} \\
\hline
\end{tabular}




\begin{tabular}{|c|c|c|}
\hline CRONOS & $\mathrm{n}_{\mathrm{e}}$ & {$[17]$} \\
\hline ETS & $\mathrm{n}_{\mathrm{e}}$ or $\mathrm{n}_{\mathrm{i}}$ & {$[13]$} \\
\hline FASTRAN & $\mathrm{n}_{\mathrm{e}}$ & {$[18]$} \\
\hline JINTRAC & $\mathrm{n}_{\mathrm{i}}$ & {$[19]$} \\
\hline RAPTOR & $\mathrm{n}_{\mathrm{e}}$ or $\mathrm{n}_{\mathrm{i}}$ & {$[15]$} \\
\hline TASK/TR & $\mathrm{n}_{\mathrm{e}}$ or $\mathrm{n}_{\mathrm{i}}$ & {$[20]$} \\
\hline TOPICS & $\mathrm{n}_{\mathrm{i}}$ & {$[16]$} \\
\hline TRANSP/PTSOLVER v.18.1 & $\mathrm{n}_{\mathrm{e}}$ & {$[14]$} \\
\hline
\end{tabular}

\subsection{Setup for benchmark of the electron and ion solvers}

The target plasma is set to be the ITER baseline scenario at the stationary plasma current flattop phase with plasma current of $15 \mathrm{MA}$, toroidal field at the magnetic axis of $5.3 \mathrm{~T}$, and geometric parameters as shown in table 2. To unify the metric coefficients in the solvers the plasma equilibrium is prescribed based on the provided EQDSK file and fixed. The impurities are prescribed to have the same profile shape as the electron density with a fixed fraction $\mathrm{nz} / \mathrm{n}_{\mathrm{e}}$ where $\mathrm{n}_{\mathrm{Be}} / \mathrm{n}_{\mathrm{e}}=0.02$ and $\mathrm{n}_{\mathrm{Ar}} / \mathrm{n}_{\mathrm{e}}=0.0005$ are assumed. The helium profile is prescribed as $\mathrm{n}_{\mathrm{He}}(\Phi)=\mathrm{n}_{0}[1-$ $\left.\left(\Phi / \Phi_{a}\right)^{2}\right]^{2}$, to reflect the peaking of the core He source due to fusion reactions, where $n_{0}=$ $0.95 \times 10^{19} / \mathrm{m}^{3}$. The neutral beam fuelling is ignored. The particle source, transport coefficients, and boundary condition are prescribed which are described in Appendix. Figure 1 shows the source, diffusivity, pinch velocity, and density profiles for the reference case calculated with ASTRA version 6 . The same source, diffusivity, and pinch velocity is applied to all the ion solvers involved in the ion solver benchmark as well as to all the electron solvers involved in the electron solver benchmark in section 3.2 . 
Table 2. Plasma geometric parameters prescribed in stationary phase

\begin{tabular}{cccccc}
\hline $\mathrm{R}_{0}(\mathrm{~m})$ & $\mathrm{a}(\mathrm{m})$ & $\kappa$ & $\delta$ & $\mathrm{Z}_{\operatorname{mag}}(\mathrm{m})$ & Volume $\left(\mathrm{m}^{3}\right)$ \\
\hline 6.20 & 1.99 & 1.85 & 0.45 & 0.50 & 819.4 \\
\hline
\end{tabular}
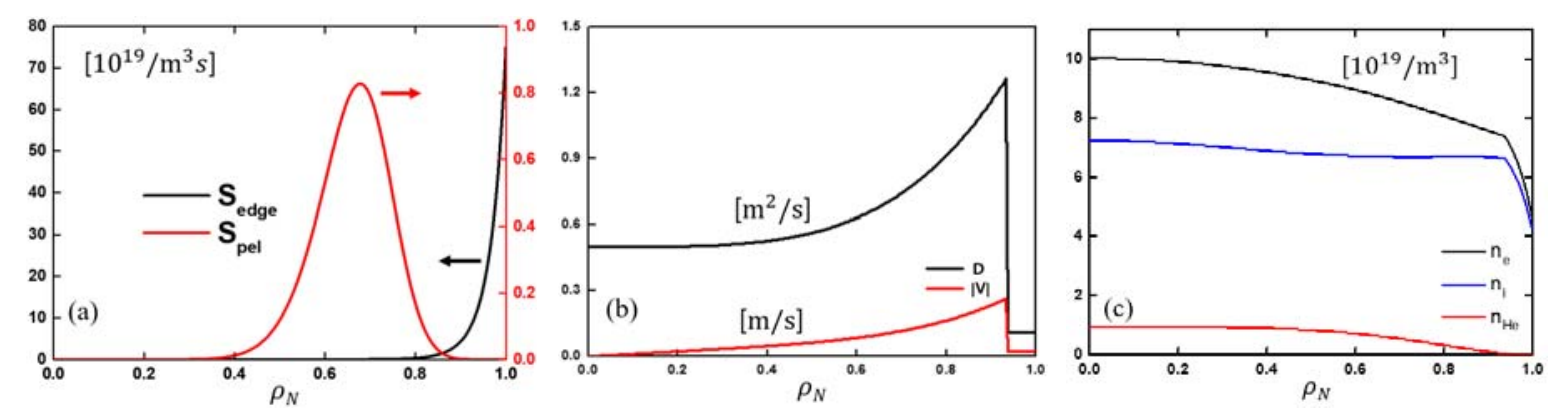

Figure 1. Edge (Sedge) and pellet (Spel) source (a), diffusivity (D) and pinch velocity $(|v|)(b)$ profiles prescribed for electron solver benchmarking in the stationary phase of the ITER baseline scenario and corresponding electron ( $\left.n_{e}\right)$, fuel ion ( $n_{i}$; deuteron + triton density), and helium density ( $\left.n_{H e}\right)$ profiles (c) calculated with ASTRA version 6 electron transport solver as reference for the prescribed impurity fractions, $n_{B e} / n_{e}=0.02, n_{A r} / n_{e}=0.0005$ and prescribed helium profile, $n_{\mathrm{He}}(\Phi)=n_{0}\left[1-\left(\Phi / \Phi_{a}\right)^{2}\right]^{2}$.

\subsection{Setup for time-varying simulations}

The target plasma is set to be the ITER baseline scenario with the total discharge time of $710 \mathrm{~s}$ composed of 0-100 s of the ramp-up phase, 100-550 s of the flattop phase, and 550-710 $\mathrm{s}$ of the ramp-down phase. The impurities are prescribed to have the same profile shape as the electron density with a fixed fraction $\mathrm{n}_{\mathrm{z}} / \mathrm{n}_{\mathrm{e}}$. The helium profile is prescribed as $\mathrm{n}_{\mathrm{He}}(\Phi)=0.109 \mathrm{n}_{\mathrm{e}}(\Phi)$. The quasi-neutrality is enforced on each magnetic flux surface. For a stable simulation, the simulation starts at $10 \mathrm{~s}$ with the initial electron density prescribed as a flat profile; $n_{e}=0.563 \times 10^{19} / \mathrm{m}^{3}$. The neutral beam fuelling is ignored. The particle source, transport coefficients, and boundary condition are prescribed in each phase of the scenario as described in Appendix. 


\section{Benchmark results}

\subsection{Particle flow balance check in the stationary phase}

Before starting the benchmark, we check the particle flow balance in each particle transport solvers at the stationary phase to see if the numerical solvers reproduce the particle conservation. As shown in equations (1)-(6), the flux and the source should make the balance in a stationary condition. It was confirmed that all the codes satisfy the flow balance. Figure 2 shows an example of the particle flow balance in ASTRA version 7 .

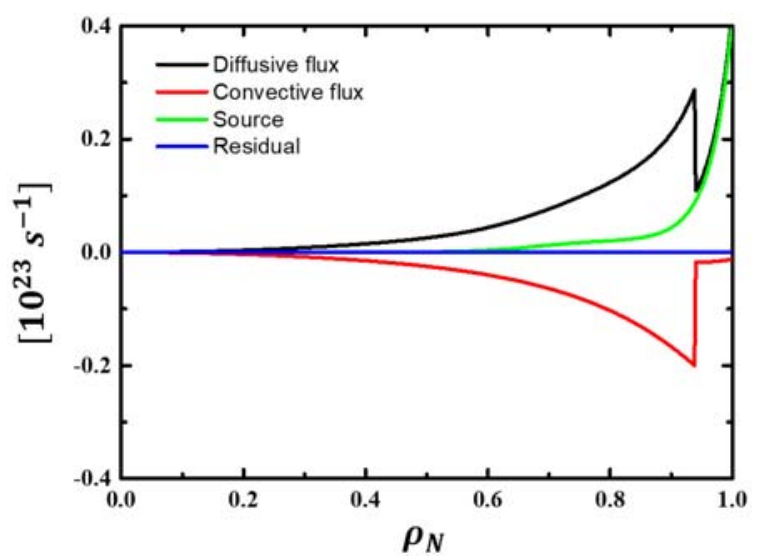

Figure 2. Particle flow balance in ASTRA v.7, where the diffusive and convective flux, source, and residual are presented in the stationary phase of the ITER baseline scenario.

\subsection{Benchmark in the stationary phase}

We start the benchmark for the ion transport solvers first in the stationary phase. The prescription of the transport coefficients and the sources described in section 2.2 are used. 

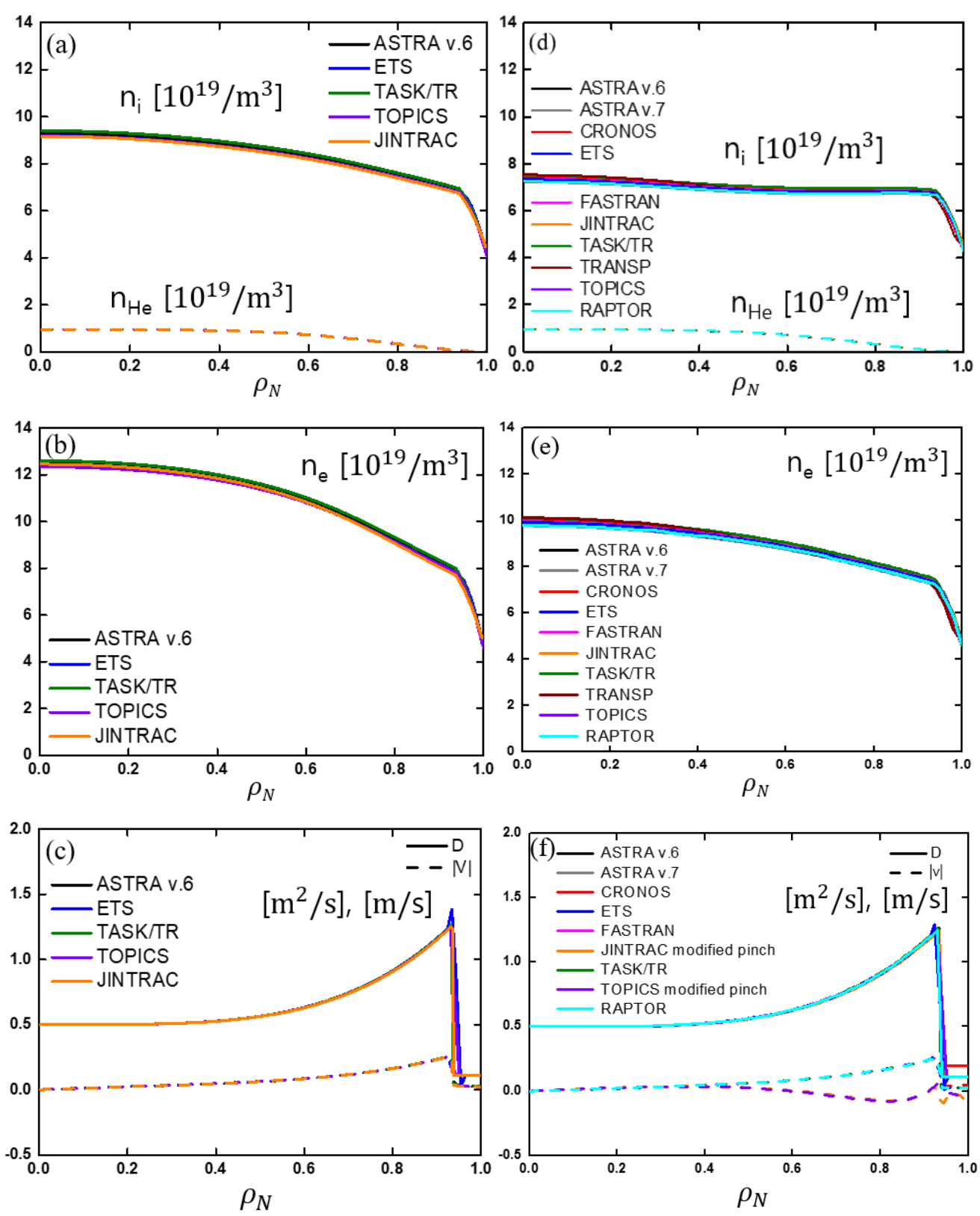

Figure 3. Particle density profiles predicted from ion (left) and electron (right) transport benchmark, respectively with the setup of simulations prescribed in section 2.2. (a),(d) fuel ion (deuteron+triton) density profiles, (b),(e) electron density profiles, (c),(f) profiles of particle diffusivities and pinch velocities in $\rho_{N}=\left(\Phi / \Phi_{a}\right)^{1 / 2}$ in the stationary phase of the ITER baseline scenario. 
Figure 3 (a) and (b) show profiles of the fuel ion density, defined as the sum of the deuteron and the triton density, and the electron density, respectively in $\rho_{\mathrm{N}}$ predicted by the ion solvers. As shown in the figures, some solvers do not reproduce accurately the jumps of the transport coefficients, $\mathrm{D}$ and $\mathrm{V}$ around the pedestal top with abrupt jumps of transport coefficients, prescribed for benchmarking, which may be related to interpolation effects at reduced grid resolution. The effect of the number of grid points will be discussed in more detail in section 4.1. In spite of this difference, all the solvers show good agreement within $2 \%$.

As described in section 2.1, the ion transport solvers can emulate the electron transport solvers by modifying the pinch term. Now, the ion solvers with these modified pinch terms are compared with the electron solvers based on the guideline described in section 2.2. Figure 3 (f) shows the difference in the pinch corrections, required for transformation to the electron flux in the ion solvers. Note that all these codes are tuned to solve the same transport equations. The profiles of the ion and the electron density and the transport coefficients are presented in figure 3 (d)-(f), respectively. The density profiles are found to agree within $3 \%$ between all the solvers regardless the type of solvers, electron or ion, and transport equations solved. Therefore, all the codes have similar numerical accuracy for identical particle fluxes.

It is noteworthy that the electron density and the fuel density profiles predicted by the ion solvers (figure 3 (a), (b)) are peaked noticeably more than those predicted by the electron solvers (figure 3 (d), (e)) for the same fuelling sources and transport coefficients, $S_{e}=S_{i}=S, D_{e}=D_{i}=D$, $\mathrm{v}_{\mathrm{e}}=\mathrm{v}_{\mathrm{i}}=\mathrm{v}$ (figure 1). This is due to the quasi-neutrality condition. The ion solvers calculate the ion density using $S_{i}=S, D_{i}=D, v_{i}=v$, then the electron density is obtained by $n_{e}=n_{i}+2 n_{H e}+18 n_{A r}$ $+4 n_{B e}$ (figure 3 (a), (b)). On the other hand, the electron solvers calculate the electron density using $\mathrm{S}_{\mathrm{e}}=\mathrm{S}, \mathrm{D}_{\mathrm{e}}=\mathrm{D}, \mathrm{ve}_{\mathrm{e}}=\mathrm{v}$, then the ion density is obtained by $\mathrm{n}_{\mathrm{i}}=\mathrm{n}_{\mathrm{e}}-2 \mathrm{n}_{\mathrm{He}}-18 \mathrm{n}_{\mathrm{Ar}}-4 \mathrm{n}_{\mathrm{Be}}$ (figure 3 (d), (e)). Therefore, both the ion and the electron density are calculated to be higher by the ion solvers than those by the electron solvers. From another point of view, the additional convective term $\delta v_{i}$ $\sim-D_{\mathrm{e}}\left(n_{\mathrm{e}}{ }^{\prime}-n_{\mathrm{i}}{ }^{\prime}\right) / n_{\mathrm{i}}$, which describes the difference between the ion and the electron fluxes (see equations (8)-(11)) can explain the difference in the ion density between the original ion solver (figure 3 (a)) and the ion solver using the modified pinch term (figure 3 (d)) for the same $S_{i}=S, D_{i}$ $=\mathrm{D}$. The reduction of the inward pinch results in a lower density for the ion solver with the modified pinch term (figure 3 (b)). This effect is generally small in present day machines but could 
be pronounceable for tokamaks with light impurities with the source peaked at the centre, like helium ash in fusion reactors. Therefore, the transport coefficients validated in present day experiments can predict different fusion performance in future machines in different types of solvers, if they are predicted by models that do not resolve differences in $\mathrm{D}_{\mathrm{i}}, \mathrm{Vi}$ vs. $\mathrm{De}$, ve. It is also noteworthy that due to the difference of the metric coefficients for diffusive and convective terms in the RAPTOR, JINTRAC, TASK/TR and TOPICS code the density decay length, $L_{n}=-R n^{\prime} / n$, depends not only on the ratio of transport coefficients, but also on the ratio of the metric coefficients. It requires additional correction for conversion from the ion to the electron solver and makes the comparison with experiments less trivial for mentioned solvers. On the other hand, the selection of metric coefficients made with these solvers is the only one for which v corresponds to the flux surface average of the orthogonal local fluxes and for which transport coefficients D and $\mathrm{v}$ are invariant with respect to the choice of the flux surface label as detailed in [11], which may be advantageous for theory-driven processing and analysis tasks.

\subsection{Benchmark of pellet fuelling modules in the stationary phase}

In parallel to the particle transport solver benchmark, we conduct the source model benchmarking. In this section, we deal with the pellet fuelling. To test the dependence of the fuel deposition on the pellet model and on the plasma parameters, we calculate the pellet deposition profile by fixing the equilibrium and kinetic profiles including the density profiles of the reference case (ASTRA v.6 in figure 1) described in section 2.2, that is no particle transport is solved here. The maximal injection speed of the intact pellets for ITER, $V_{\text {pel }}=300 \mathrm{~m} / \mathrm{s}$ is chosen for simulations [4]. A normal injection at the mid-plane is assumed and no edge puffing is applied in the simulations. We evaluate the pellet deposition profiles for 1) HFS injection assumed for plasma fuelling and LFS injection assumed for ELM pacing by pellets, for 2) small and large pellets with $\mathrm{V}_{\mathrm{p}}=33 \mathrm{~mm}^{3}$, the minimal size required for ELM pacing, and $90 \mathrm{~mm}^{3}$, the maximal size foreseen in ITER, respectively. We also carried out the sensitivity studies on plasma parameters of the pedestal and the separatrix by varying the pedestal temperature (20\% higher and lower than the reference), pedestal density ( $20 \%$ higher and lower than the reference), separatrix temperature (100\% higher and lower than the reference), and separatrix density (20\% higher and lower than the reference). For this benchmark study, ASTRA with SMART [22] and JINTRAC with HPI2 [23] are employed for simulating the 
pellet fuelling. Both models simulate the pellet ablation and further drift of ablated particles toward LFS.

The results are presented in figure 4. As shown, JINTRAC with HPI2 predicts deeper deposition of the pellet particles compared to ASTRA with SMART in all cases of the HFS injection meanwhile the qualitative dependencies look similar. This result agrees with the previous study [24]. The sensitivity scan of various parameters reveals that the HFS injection provides much deeper particle source than the LFS injection due to the drift of the ablated pellet particles toward the plasma centre making the HFS injection much more efficient for fuelling (see figure 4 (a), (b)). The larger pellets, $\mathrm{V}_{\mathrm{p}}=90 \mathrm{~mm}^{3}$ produce about $\sim 2-2.5$ times higher peaked deposition profile with deeper penetration than smaller ones, $V_{p}=33 \mathrm{~mm}^{3}$ as shown in figure 4 (a) and (b). The pedestal temperature has stronger impact on the particle deposition than the pedestal density with both models because of higher pressure of the plasmoid produced by pellet $[22,23]$ which depends mostly on the target plasma temperature at the location of pellet ablation and a much smaller extent on the background density. As a result, the predicted drift displacement and particle deposition is more strongly affected by a variation in $\mathrm{T}_{\text {ped }}$ as compared to $\mathrm{n}_{\text {ped. }}$. The depth of deposition increases with increase of the pedestal temperature (see figure 4 (c)). The relative increase of the maximum of particle deposition profile predicted by SMART is similar to HPI2 predictions, but the impact on the final distribution is much less pronounced because the absolute deposition depth predicted by SMART is noticeably smaller. The separatrix density and the temperature do not affect the deposition profile for the changes enforced in this study. Note that both models predict similar depth of the pellet ablation for each of the pellet size. For both the 33 and $99 \mathrm{~mm}^{3}$ LFS and HFS pellets in the process of ablation penetrate to the top of pedestal, i.e. sufficiently deep to trigger ELMs. For LFS pellets the SMART and HPI2 models predict almost full removal of ablated particles due to the outward drift even for small pellets as shown in figure 4 (b), which agrees with the assumptions of the integrated analysis [4]. It makes possible to provide the ELM pacing from the LFS pellet injection independent on the density control by HFS pellet injection [4]. 

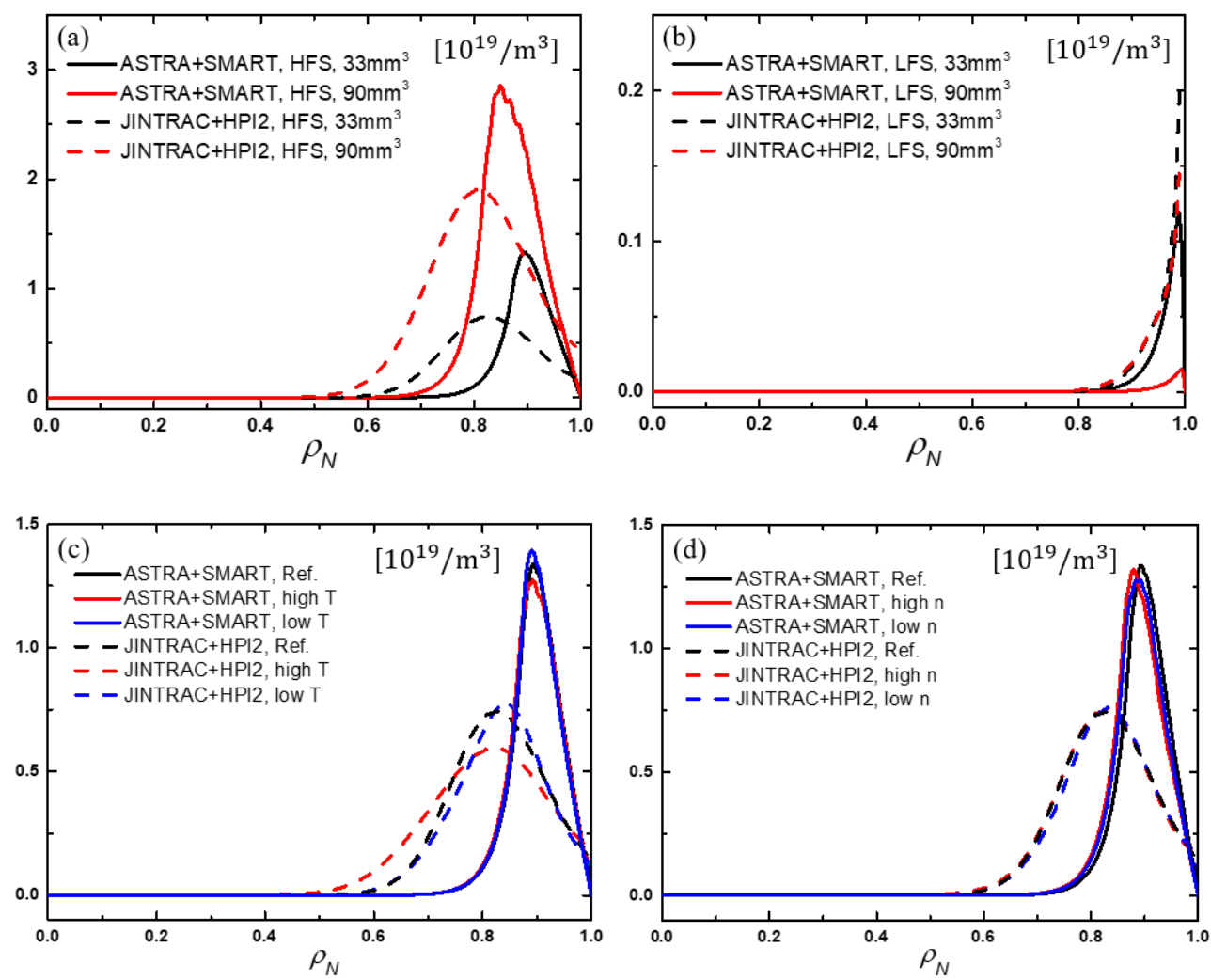

Figure 4. Particles deposition profiles predicted by ASTRA with SMART (sold lines) and JINTRAC with HPI2 (dashed lines) for (a) HFS injection with pellet volume of $33 \mathrm{~mm}^{3}$ and 90 $\mathrm{mm}^{3}$, (b) LFS injection with pellet volume of $33 \mathrm{~mm}^{3}$ and $90 \mathrm{~mm}^{3}$, (c) various pedestal temperatures, (d) various pedestal densities with HFS injection with $V_{p}=33 \mathrm{~mm}^{3}$ in $\rho_{N}=\left(\Phi / \Phi_{a}\right)^{1 / 2}$ in the stationary phase of the ITER baseline scenario.

\subsection{Benchmark of gas fuelling modules in the stationary phase}

In this section we conduct benchmarking of gas fuelling modules to evaluate uncertainties in source predictions obtained by reduced models that are commonly in use for integrated modelling, and to assess sensitivities of the source profile predictions with respect to uncertainties in background plasma conditions in the ITER baseline scenario and with respect to details in model assumptions. Here, the particle sources caused by penetration of the neutrals from the edge are referred as "cold neutral sources" for historical reasons, when in the limiter tokamaks the atomic hydrogen isotopes came directly from the molecules to the plasma core with the molecule dissociation energy $(\sim 2-5$ 
$\mathrm{eV}$ ). In the diverted tokamaks of the reactor scale, most part of the neutrals in the confined region are the results of the process of charge exchange of the original "wall" neutrals with plasma ions [25], which is most significant in the plasma edge region, while the neutral background is extremely small in the plasma centre, with neutrals being only created there through the process of recombination of plasma ions and electrons. The recombination determines the density of neutral gas in the core, but being practically compensated by ionisation does not affect the total particle source in the core. Due to the nature of the process of their formation, the average energy for the majority of neutrals in the confined region is close to the local ion temperature. Here, the "cold" neutrals are defined as the sum of neutrals penetrated from SOL and neutrals produced from charge exchange with the ions and the process of recombination of the thermal fuel ions with electrons. Simulations have been carried out with ASTRA [11] and JINTRAC [19] in interpretive mode for the ITER baseline scenario in stationary L-mode and H-mode conditions. For L-mode, parabolic density and temperature profiles are prescribed with axial and boundary densities and temperatures that are in line with expectations for the end of the current ramp-up phase with Ohmic heating at $\mathrm{I}_{\mathrm{p}}$ = 15 MA [26] (see figure 5 (a)). For H-mode, the density and temperature profiles are used in agreement with predictions from recent integrated transport modelling studies such as $[4,26]$, where pedestal conditions are determined by scaling derivations from simulation scans with the EPED1 and SOLPS codes for the ITER baseline conditions [28] (see figure 5 (b)). For simplicity of benchmarking, impurities are not considered in the calculations and the plasma is assumed to consist of deuterium only so to neglect isotope effects such as isotopic segregation due to the isotope dependent inertia of the neutrals and desegregation caused by interspecies charge exchange reactions. A fixed cold neutral influx is imposed at the plasma boundary. Cold neutral sources have been calculated with the NEUT code implemented in ASTRA [11] as well as with FRANTIC [29] and EIRENE [30] which are both available in JINTRAC. While EIRENE is a kinetic Monte Carlo code which can calculate neutral transport for the actual flux surface geometry with high accuracy but at the cost of increased computational time, much faster but simplified models for the calculation of cold neutral sources are employed with NEUT and FRANTIC. The main differences in neutral model assumptions for these codes are summarised in table 3. Besides the differences listed in the table there is also difference in the ionisation, charge exchange, and recombination cross-sections used in the modelling. The ionisation cross-sections are taken from [32] in NEUT and from [33] for both EIRENE and FRANTIC. The charge exchange cross-section, $\sigma_{C X}$ is 
determined in the original version of FRANTIC on basis of a scaling given in [34], while crosssection estimates in NEUT and EIRENE are derived from [31] and [33], respectively. For typical ion temperatures at the edge region in a range $\sim 0.1-3.0 \mathrm{keV}$, the difference in calculated crosssection reactivities was found to remain below $\sim 25 \%$, i.e. the effect of varying assumptions for $\sigma_{\mathrm{CX}}$ may be small. To scan the sensitivity to pedestal and boundary conditions, the ITER H-mode and L-mode simulations have been repeated with varied assumptions for the temperature and density at the top of the pedestal and at the separatrix.

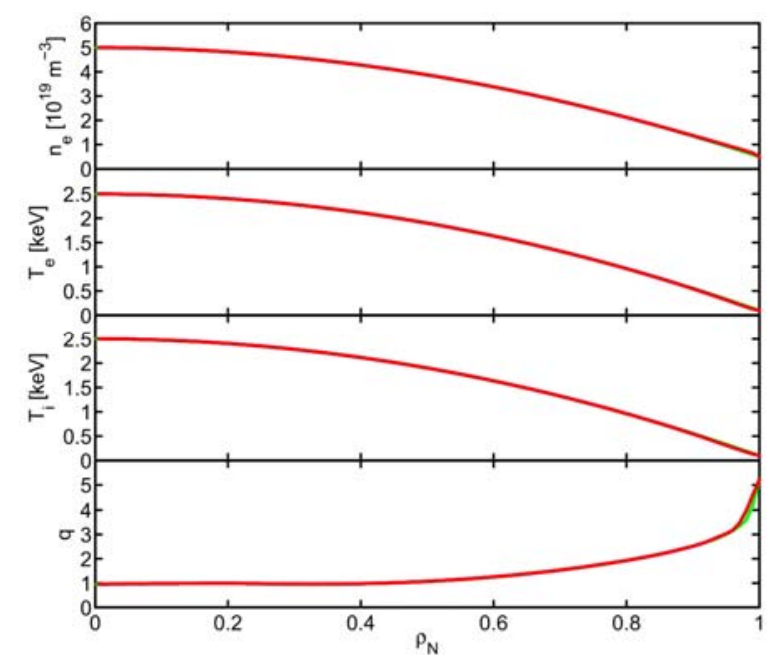

(a)

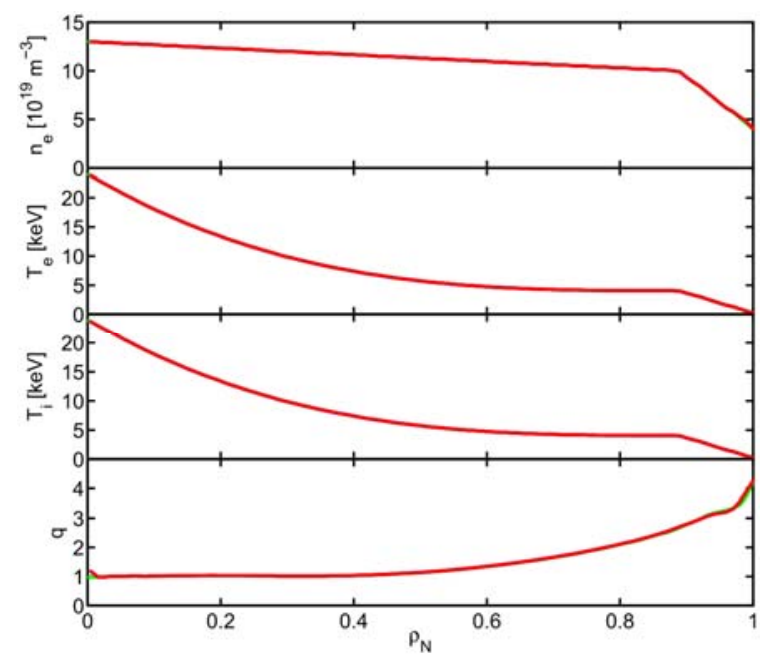

(b)

Figure 5. From top to bottom: Profiles of electron density, electron temperature, ion temperature, and safety factor for the ITER baseline 15 MA 5.3 T stationary L-mode (a) and Hmode (b) configurations considered for the cold neutral source predictions.

Table 3. Differences between the three cold neutral source codes (NEUT, FRANTIC, and EIRENE) in standard model assumptions and conditions as used for the simulations of cold neutral profiles for the ITER L-mode and H-mode plasmas. 


\begin{tabular}{|c|c|c|c|}
\hline & NEUT & FRANTIC & EIRENE \\
\hline Model scheme & $\begin{array}{l}\text { Iterative solution of } \\
\text { discretised 1-D kinetic } \\
\text { equation for neutral } \\
\text { distribution }\end{array}$ & $\begin{array}{l}\text { Solution of discretised } \\
\text { integral equation for } \\
\text { neutral source density }\end{array}$ & $\begin{array}{l}\text { 3-D Monte Carlo } \\
\text { solution for linear } \\
\text { Boltzmann equation } \\
\text { for neutral transport }\end{array}$ \\
\hline Geometry & Slab, thickness $2 \times_{\mathrm{a} 0}$ & $\begin{array}{l}\text { Cylindrical, } \\
\text { radius } r=\rho_{\text {tor,sep }}\end{array}$ & $\begin{array}{l}\text { 2-D flux surface } \\
\text { geometry from Grad- } \\
\text { Shafranov solver }\end{array}$ \\
\hline $\begin{array}{l}\text { Neutral energy } \\
\text { distribution }\end{array}$ & $\begin{array}{l}\text { Average energy over } \\
100 \text { charge exchange } \\
\text { neutral generations }\end{array}$ & $\begin{array}{l}\text { Set of distinct energy } \\
\text { levels determined by } \\
\text { energy of neutral } \\
\text { influx, number of cells } \\
\text { and } T_{i} \text { in each cell }\end{array}$ & Complete spectrum \\
\hline $\begin{array}{l}\text { Neutral velocity } \\
\text { distribution at the } \\
\text { plasma boundary }\end{array}$ & $\begin{array}{l}\text { Orthogonal to } \\
\text { boundary surface }\end{array}$ & Isotropic & $\begin{array}{l}\text { Isotropic (with current } \\
\text { implementation in } \\
\text { JINTRAC) }\end{array}$ \\
\hline
\end{tabular}

The results of simulations by three codes listed in the table for profiles displayed in figure 5 at the L-mode and H-mode phases are presented in figure 6. As shown, significant variations in the neutral penetration depth are observed in both the L-mode and the H-mode case. As expected, the neutral source profiles are much wider for the L-mode case, while the neutrals are mainly ionised in the outermost edge region in the H-mode case. The ionisation source from the cold neutrals is located at the outer $10-20 \%$ of the minor plasma radius for the L-mode, and $5-10 \%$ in the H-mode operation. Nevertheless, the fraction of neutrals that are ionised inside the pedestal could be as large as $\sim 10-20 \%$ according to NEUT and EIRENE predictions. The uncertainty for the percentage of cold neutrals that are ionised inside the core region is considerable not only due to variations in predictions obtained by different neutral codes, but also due to uncertainties in pedestal conditions. 
For a variation of pedestal or separatrix temperatures and densities within a factor $\sim 2$, the integrated net ionisation source at the top of the pedestal varies between $\sim 10$ and $40 \%$ of the total net ionisation source in the confined region according to NEUT and EIRENE predictions. For FRANTIC, predictions for the integrated ionisation source are typically lower because neutrals are predicted to penetrate less deeply into the core with FRANTIC. As a consequence, the fraction of cold (or charge exchanged) neutrals from the edge that are ionised in the core is negligible. The net ionisation source in the core is determined by a balance between plasma ion recombination and reionisation processes. As a general trend, the penetration depth of cold neutrals might be overestimated with NEUT and underestimated with FRANTIC compared to the EIRENE predictions.

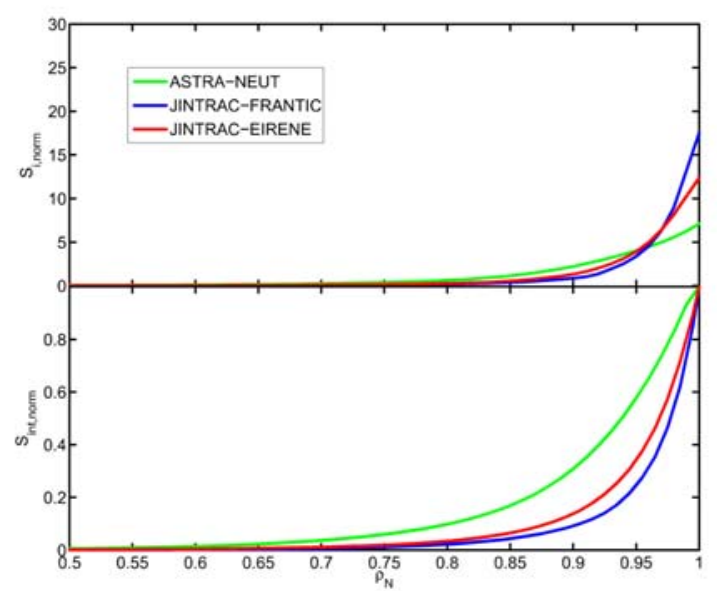

(a)

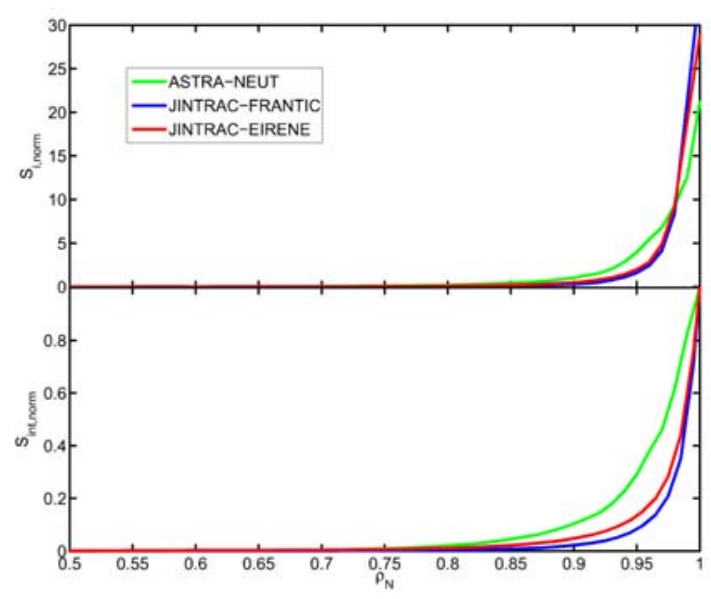

(b)

Figure 6. Profiles for the normalised ionisation source rate $S_{i, \text { norm }}=$ $S_{i}(\rho) \cdot V\left(\rho_{\text {sep }}\right) / \int_{\rho^{\prime}=0}^{\rho_{\text {sep }}} S_{i}\left(\rho^{\prime}\right) \frac{d V\left(\rho^{\prime}\right)}{d \rho^{\prime}} d \rho^{\prime}$ (top) and integrated net ionisation source rate $S_{\text {int,norm }}(\rho)=\int_{\rho^{\prime}=0}^{\rho} S_{i}\left(\rho^{\prime}\right) \frac{d V\left(\rho^{\prime}\right)}{d \rho^{\prime}} d \rho^{\prime} / \int_{\rho^{\prime}=0}^{\rho_{\text {sep }}} S_{i}\left(\rho^{\prime}\right) \frac{d V\left(\rho^{\prime}\right)}{d \rho^{\prime}} d \rho^{\prime}$ (bottom) as predicted by the cold neutral codes NEUT (green), FRANTIC (blue), and EIRENE (red) for the ITER baseline 15 MA

5.3 T stationary L-mode (a) and H-mode (b) configurations as illustrated in figure 5. 
The differences in the lengths of decay of the ionisation sources at the edge can be explained by the difference in the "effective radii" for neutrals penetrated from the edge in different codes. In general the influx of the neutrals through the separatrix is three-dimensional. Even in the case of toroidal symmetry the influx is not homogeneous in the poloidal direction as well as the plasma temperature and density along the separatrix. In FRANTIC, the influx is poloidally symmetric, thus the normalised ionisation length is proportional to $1 / \rho_{\mathrm{a}}$, where $\rho_{\mathrm{a}}$ is $\rho$ at the separatrix, $\rho_{\mathrm{a}}=\left(\Phi_{\mathrm{a}} / \pi \mathrm{B}_{0}\right)^{1 / 2}$. For benchmarking the poloidal distribution of the neutral influx in the EIRENE simulations was assumed symmetric. The efficient ionisation length appeared to be lower than in the NEUT case. In the NEUT solver it is assumed that the maximal neutral flux corresponds to the mid-plane, thus the normalised ionisation length is proportional to $1 / \mathrm{a}$. Therefore, the radial decay of the ionisation source from the separatrix predicted by the NEUT code is $\rho_{a} / a$ times wider than predicted by the FRANTIC code. For the ITER case it corresponds to $\rho_{a} / a \sim 1.3$, which is quantitatively close to the result of simulations.

Comparing cold neutral source predictions obtained with neutral codes coupled to 1.5-D transport codes for the confined region with results obtained in integrated core-edge-SOL simulations e.g. from SOLPS-ITER [35] or JINTRAC [19] in which neutral transport is calculated for the entire domain inside the vacuum vessel (or for the main region of interest excluding only the plasma centre where neutral interactions are insignificant) for identical background plasma configurations, it is found that deviations are essentially due to simplified assumptions for boundary conditions defining the properties of incoming neutrals at the separatrix that need to be specified for neutral codes in simulations that are restricted to the confined region. While the poloidal distribution of neutrals entering the confined region may be highly asymmetric in fully integrated core-edge-SOL simulations, a poloidally symmetric distribution needs to be set up for the neutral codes with simplified geometry assumptions. In case of a poloidally localised neutral influx, the integrated normalised cold neutral source on top of the pedestal was found to vary by $\sim 50 \%$ as a function of the poloidal location of the penetration of neutrals in EIRENE predictions that are limited to the confined region for ITER H-mode scenario conditions as shown in figure 5 (b). A significant variation in the width of the neutral ionisation source in the confined region is predicted due to a strongly varying cold neutral penetration depth as a function of $\rho$ that is caused by a significant poloidal dependence in $|\nabla \rho|$ near the plasma boundary, with deepest penetration achieved on the LFS near the mid-plane and minimum penetration predicted near the X-point. Near 
the X-point, the fuelling efficiency may also be reduced due to an increased escape probability for charge exchanged neutrals. In addition to the uncertainty caused by the poloidal dependency of the neutral influx, deviations in assumptions for the velocity distribution of the inflowing neutrals may also need to be considered. For neutral codes applied in simulations for the confined region only, the latter is typically assumed to be isotropic, mono-energetic and independent of the poloidal location, while that is not necessarily the case in fully integrated core-edge-SOL simulations. As an example, the integrated normalised cold neutral source on top of the pedestal is predicted to vary by $\sim 20 \%$ in EIRENE simulations of the ITER H-mode conditions as illustrated in figure 5 (b) with varying assumptions for the velocity distribution of the incoming neutrals (comparing isotropic vs. delta distributions).

\subsection{Benchmark in time-evolving plasma}

For the second phase of benchmarking, we solve the particle transport in the entire discharge of the ITER baseline scenario, including the L-mode ramp-up phase, L-H transition, flattop H-mode phase, H-L transition, and L-mode ramp-down phase. The ion and the electron solvers are benchmarked together with prescribed evolutions of the plasma configuration, particle transport coefficients, sources, and so on as described in Appendix.

The benchmark results are shown in figure 7 where ASTRA v.6, v.7, TOPICS, and JINTRAC are involved. They present good agreements within $6 \%$. The profiles are compared in figure 8 for 6 different phases of the scenario. The most outstanding difference is originated from the pedestal density, particularly in the ramp-down H-mode phase. In the following sections, we will try to address the possible origins of the differences between code predictions observed in the benchmarking. 


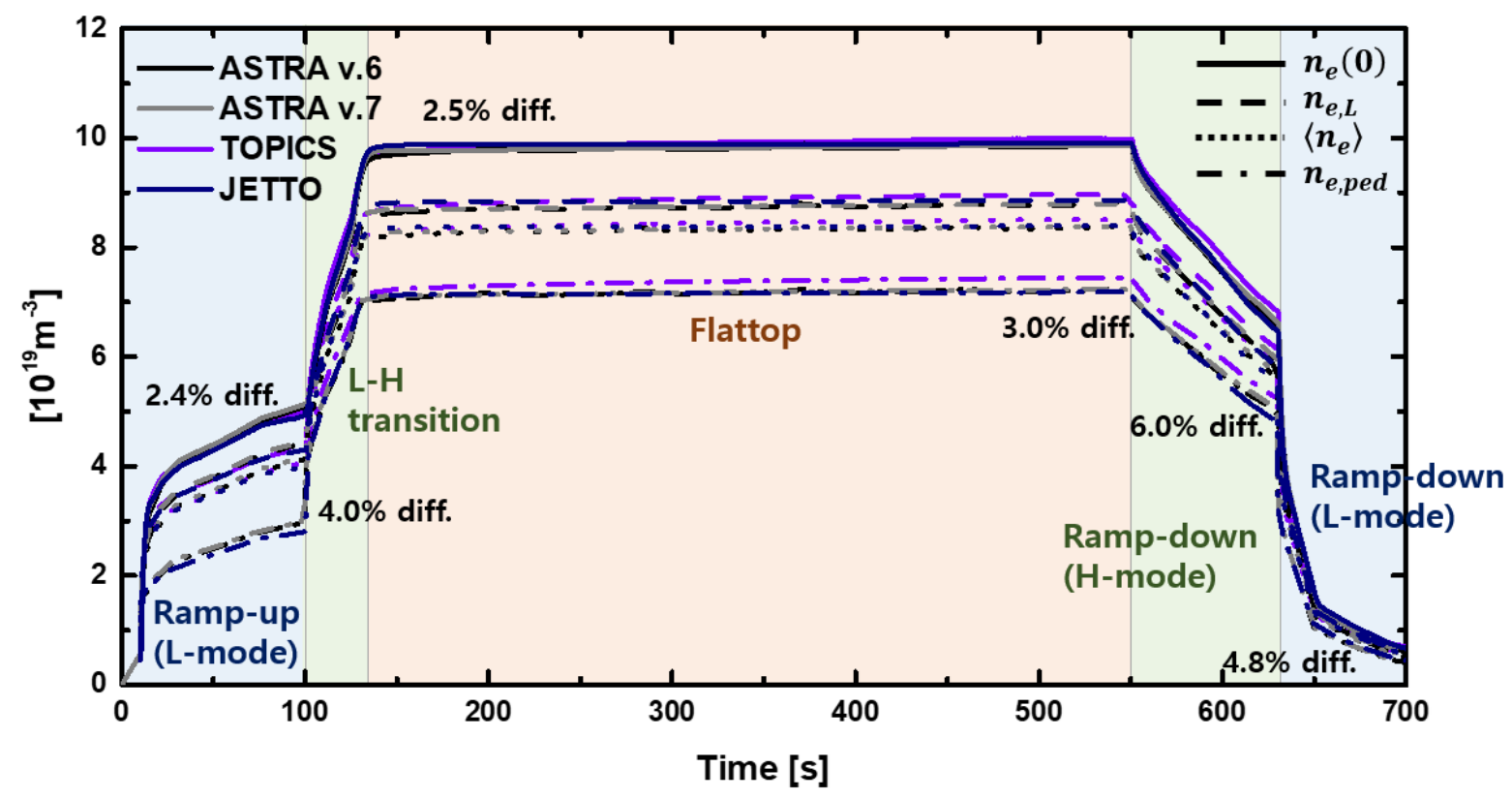

Figure 7. Time evolution of the central density, line-averaged density, volume averaged density, and pedestal density for electrons. The differences are indicated in each phase of the scenario; Ramp-up (L-mode), L-H transition, Flattop, Ramp-down (H-mode), Ramp-down (L-mode).
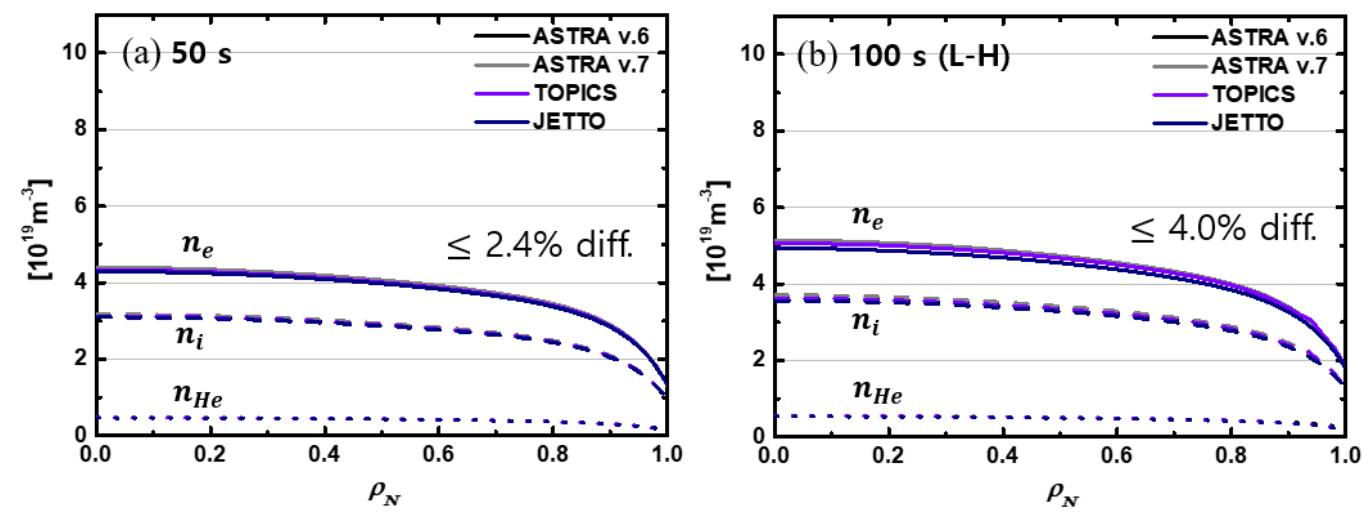

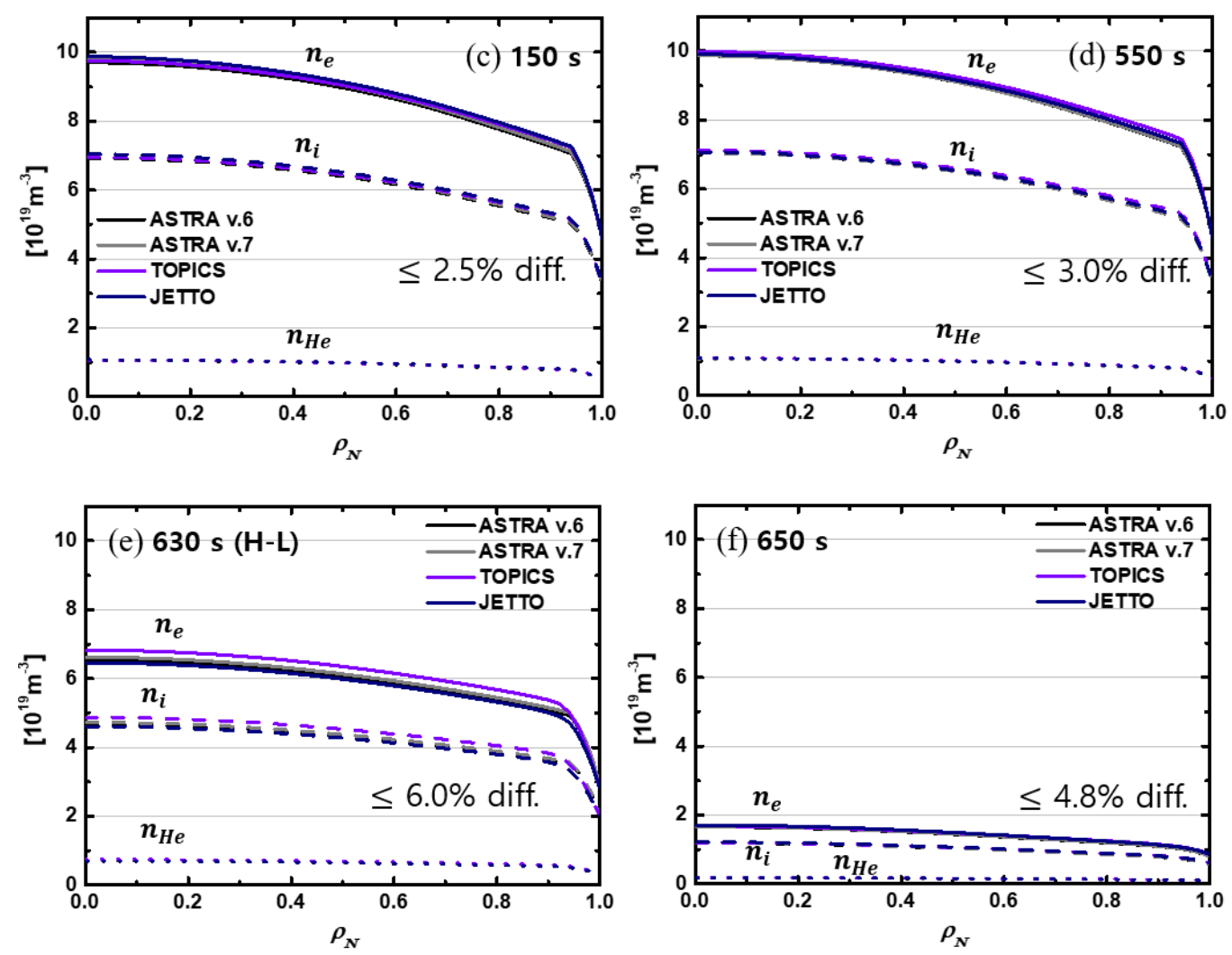

Figure 8. Particle density profiles predicted for benchmark of time-evolving plasmas with the setup of simulations prescribed in section 2.3 at $50 \mathrm{~s}$ in the ramp-up L-mode (a), at $100 \mathrm{~s}$ at the $L-H$ transition (b), at $150 \mathrm{~s}$ right after the $L-H$ transition (c), at $550 \mathrm{~s}$ at the end of the current flattop (d), at $630 \mathrm{~s}$ at the H-L transition (e), and at $650 \mathrm{~s}$ during the ramp-down L-mode (f) phase of the ITER baseline scenario.

\section{Discussion}

We discuss the possible reasons for the discrepancies between particle transport solvers observed during the benchmark; 1) difference in mapping of the EQDSK data on individual code's grids equilibrium, 2) difference in grid resolution affecting accuracy for reproduction of transport coefficients, as mentioned in section $3.2,3$ ) type of radial coordinate used in the solver and difference in accuracy of the particle source approximation due to difference in the radial grid distribution, 4) volume evolution term. Then, the impact of the integrated plasma performance on the particle transport will be discussed in this section. 


\subsection{Origins of the discrepancies in transport code predictions}

\section{Equilibrium}

Note that for the benchmark we provided the equilibrium for a certain time slices in the format of EQDSK. In the individual simulations by different solvers in the benchmark, the EQDSK data from the 2-D equidistant $(\mathrm{R}, \mathrm{Z})$ grid are converted somehow to the 1-D profiles related to the magnetic surfaces. Some solvers convert the equilibrium on their individual grid directly, some solvers use just the core boundary from the EQDSK file and recalculate the equilibrium consistently with the simulated pressure and current density profiles. Unfortunately, even the codes, which use the EQDSK directly, do not renormalise the plasma volume provided in the EQDSK file. Both can affect the discrepancy of the results of benchmarking as shown in figure 9 (a) and (b), where the enclosed volume in each flux surface is compared for the ion solver benchmark and the electron and modified ion solver benchmark, respectively. The discrepancy can affect the total integrated particle source as well as the number of particles in the plasma volume.
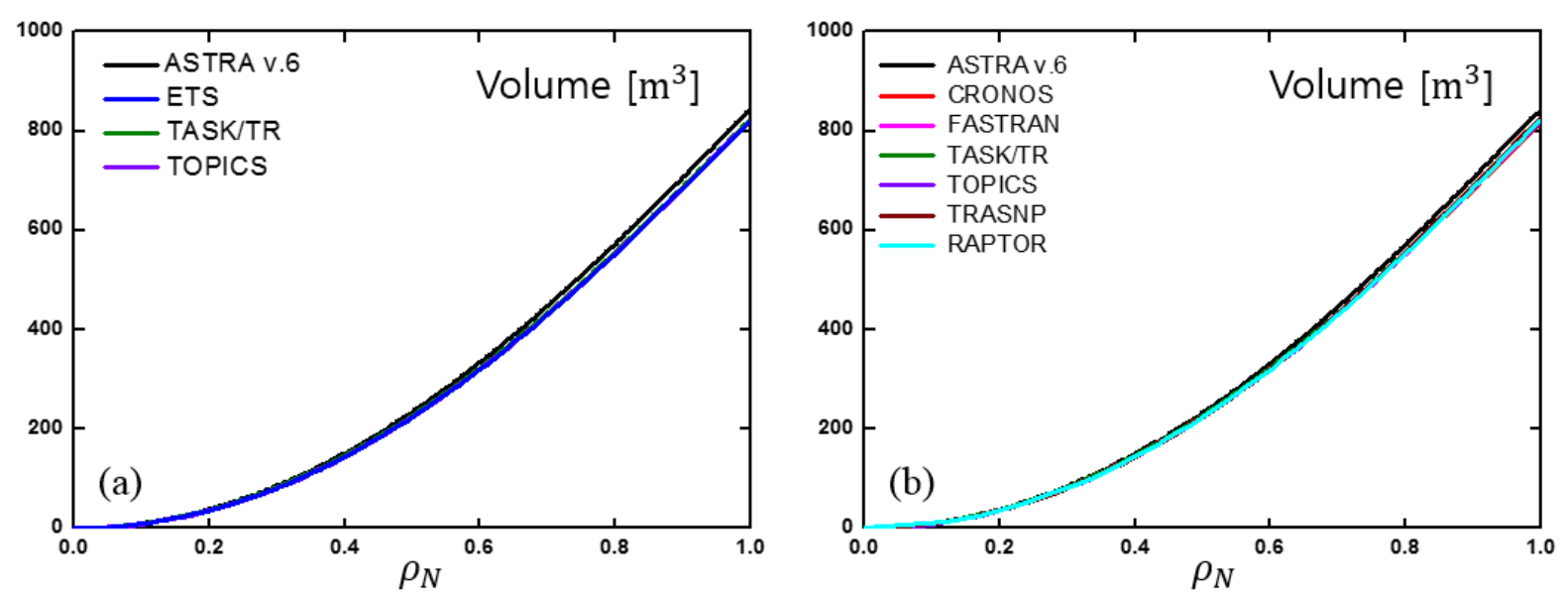

Figure 9. Comparison of enclosed volumes for the ion particle transport solver benchmark (a) and electron and modified ion particle transport solver benchmark (b) in the stationary phase. 


\section{Grid resolution}

As shown in section 3.1, sparse radial grids cannot reproduce accurately the jumps of the transport coefficients, D and V around the pedestal top with abrupt jumps of transport coefficients, prescribed for benchmarking. This influences the fuelling rate, the number of the He particle, and in particular the location of the pedestal, etc. For example, although we set the position of pedestal top to $\rho_{\mathrm{N} \text {,ped }}=\sqrt{0.88}$, the real pedestal position in the numerical calculations is practically assigned to the grid point closest to $\rho_{\mathrm{N}}=\sqrt{0.88}$. This makes the difference among the simulations with the different resolution of grid, especially due to the steep density gradient in the pedestal region, as shown in figure 11 (a). This effect is shown in time-evolution simulations as well. Figure 11 (b) shows the density profile at $\mathrm{t}=150 \mathrm{~s}$ of the time-evolving plasma benchmarking in section 3.5, which is a time after L-H transition. The difference in the pedestal location is clearly seen and causes about $\pm 2 \%$ difference at the centre. Note that the number of grid points in the time-evolution benchmark in section 3.5 is as follows; 280-308 in ASTRA v.6 (the number of grid points is adjusted while the plasma volume evolves), 381 in ASTRA v.7, 51 in TOPICS, and 200 in JINTRAC.
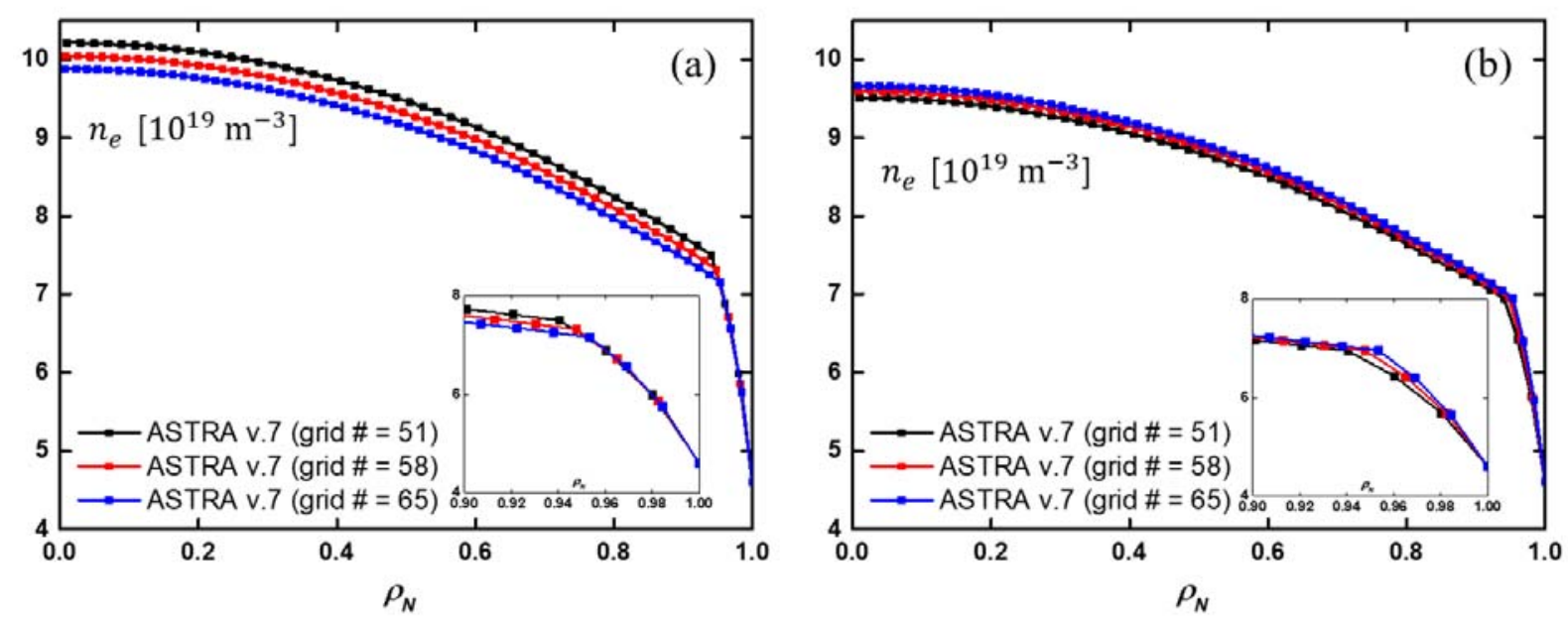

Figure 11. Electron density profiles with different grid numbers $(51,58,65)$ for stationary (a) and time-evolving plasma (at $150 \mathrm{~s}$, after $L-H$ transition) (b). The pedestal region of $\rho_{N}=0.90$ 1.00 zoomed in is inserted in each figure. 


\section{Radial coordinate and grid distribution}

The particle transport equation is usually solved either in the radial coordinate of $\rho$ or $\rho_{N}$ as shown in equation (1)-(7). For example, $\rho$ and $\rho_{N}$ are used in ASTRA v.6 and ASTRA v.7, respectively. For the solvers using $\rho$, the initial grid is distributed uniformly while starting the simulation, then as the plasma volume evolves, the number of nodes is adjusted to the evolving flux with the variable size of the edge cell $\delta \rho_{\text {edge }}$, the distance between the last two grids at the edge, while keeping the same grid distribution inside the boundary region. Therefore, the numerical approximations of the differential operator and particle source can oscillate together with the size of the edge cell. On the other hand, the number of grid points can be kept constant and the grid is distributed uniformly regardless of the equilibrium evolution for the solvers using $\rho_{N}$. Therefore, the density evolution in time can be different between the solvers using $\rho$ and $\rho_{N}$, respectively via this grid distribution at the edge.

To investigate this effect in more detail, we designed two specific cases where the plasma volume expands within $50 \mathrm{~s}$ and $100 \mathrm{~s}$, respectively as follows; triangularity from 0.00 to 0.45 , elongation from 1.0 to $1.8, \rho_{a}$ from 2.056 to 2.797 in the reference ITER baseline scenario. We solve the electron particle transport with prescribed D, v, particle source, boundary conditions, and equilibrium. The calculated electron density is compared between solvers using $\rho$ and $\rho_{N}$. In this work we employed ASTRA v.6 and v.7 for them, respectively. Note that here we intentionally decreased the number of the grid points from typical used in ASTRA $(\sim 100-500)$ to those used in sparse grid solvers $(\sim 50)$ for ASTRA v.6 to amplify this effect. They present different behaviour as shown in figure 12. In the solver with $\rho$, oscillations occur during the expansion of the plasma configuration and the calculated density is different from that in the solver with $\rho_{N}$. The oscillations in the solver with $\rho$ are found to be originated from the grid distribution at the boundary. Figure 12 (c) shows the time evolution of the normalised edge cell size $\left(\delta \rho_{\text {edge }}\right)$ to the central cell size $\left(\delta \rho_{0}\right)$. The oscillation is clearly seen. As the plasma volume increases $\delta \rho_{e d g e}$ increases and, if it becomes too large, $\delta \rho_{\text {edge }}>1.8 \delta \rho_{0}$, a new regular grid point is added and a new edge cell is assigned so that $\delta \rho_{\text {edge }}=\delta \rho_{\text {edge,old }}-\delta \rho_{0}$. 

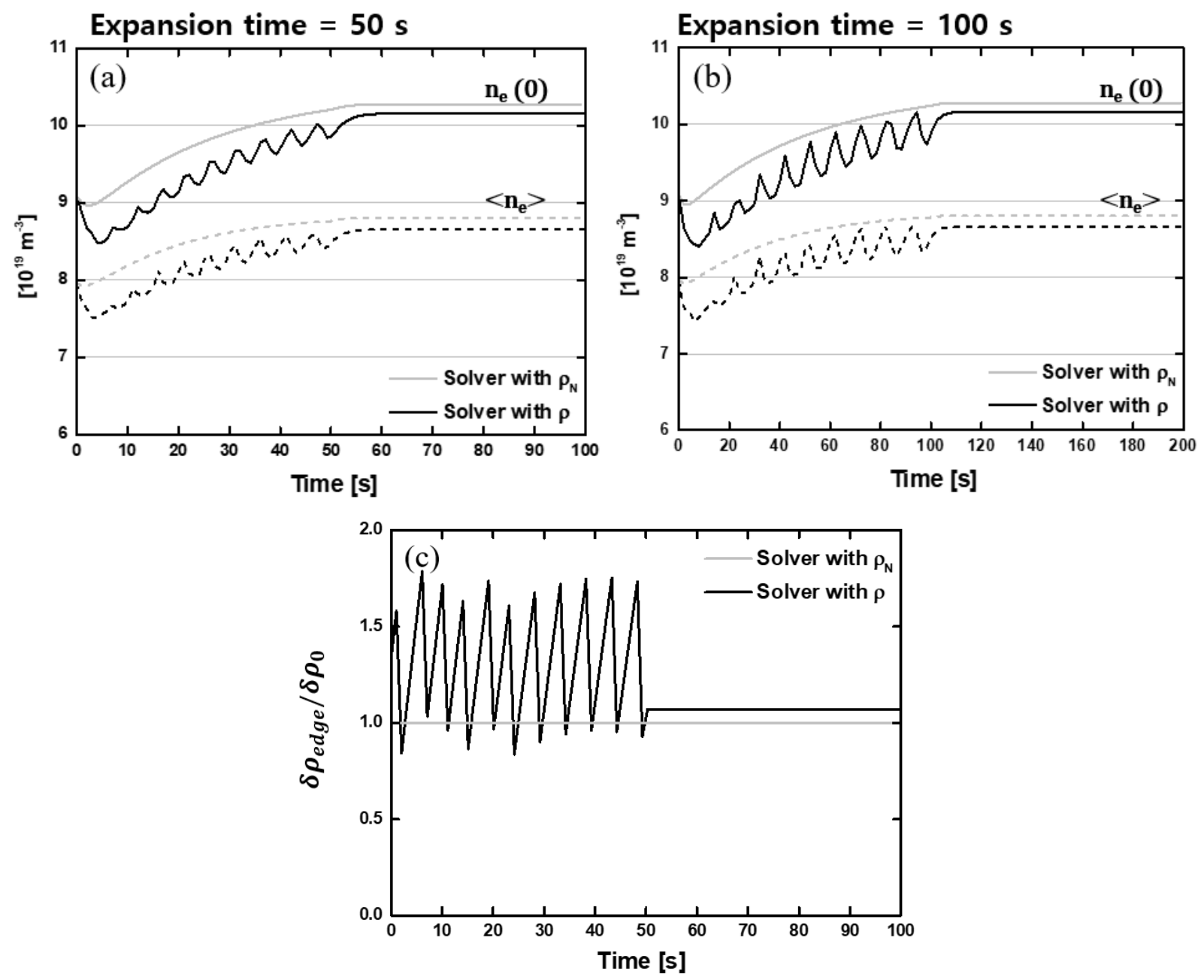

Figure 12. Time evolution of central electron density, $n_{e}(0)$ and volume-averaged electron density, $<n_{e}>$ of the solvers using $\rho$ and $\rho_{N}$ for the case with the plasma volume expansion up to $50 s(a)$ and $100 s(b)$. (c) Time evolution of the normalised edge cell size ( $\left.\delta \rho_{\text {edge }}\right)$ to the central cell size $\left(\delta \rho_{0}\right)$.

Now, we discuss the effect of this grid distribution on the results of the particle transport simulation. Analysing the density oscillation phase, we found that the source and the diffusion profiles are varying significantly while satisfying the particle flow balance. The edge grid variation causes the integrated source profile variation and the variation of the grid at the steep gradient region of the pedestal causes the diffusion flux variation. These variations of the source and 
diffusion profiles result in oscillating $\frac{d n}{d t}$. Figure 13 shows these variations of the integrated source, diffusion flux, convective flux, and $\frac{d n}{d t}$ in an oscillation phase, 26-30 s of the ion solver with $\rho$ in figure 13 (a). Depending on these variations of the integrated source and the diffusive flux, $\frac{d n}{d t}$ can have negative, zero, and positive signs which results in oscillation of the density evolution as shown in figure 13.
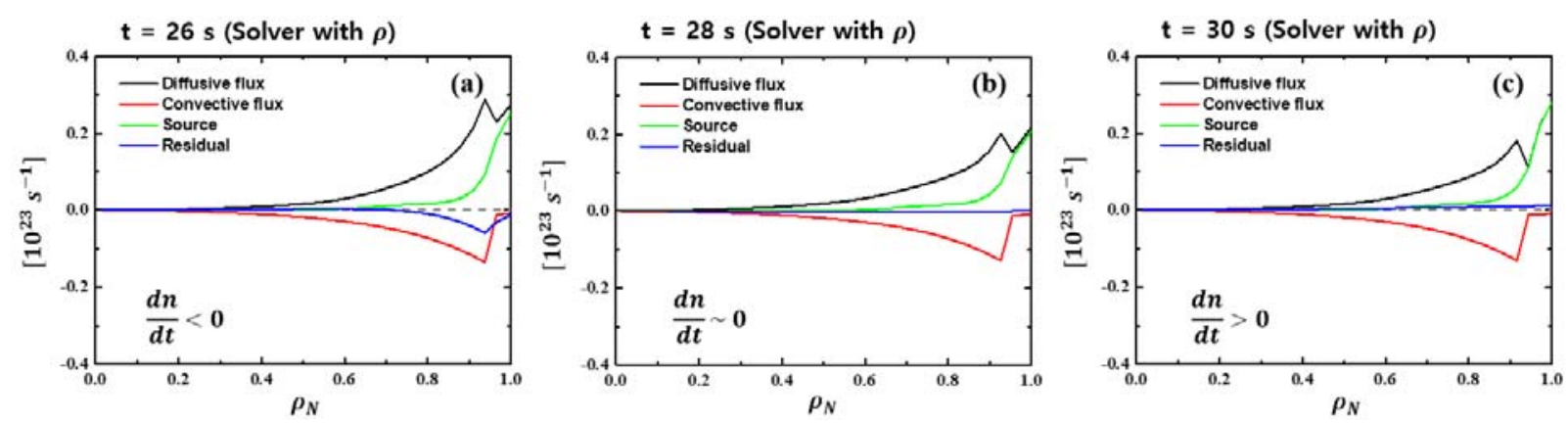

Figure 13. The variation of the integrated source, diffusion flux, convective flux, and sign of $\frac{d n}{d t}$ at $26 s(a), 28 s(b)$, and $30 s(c)$ in an oscillation phase of the solver with $\rho$ in figure $12(a)$. The sign of $\frac{d n}{d t}$ is negative, zero, and positive, respectively.

Conservative finite element schemes reflect the particle conservation law of real physical processes, but for numerical integrals on the chosen grid rather than the analytical integrals of the sources. That is caused by low accuracy of the Simpson approximation for the edge sources with exponential decrease at the edge, but does not affect much the smooth sources in the core plasmas. This numerical effect can produce large difference in the simulations of the L-mode phases with a dominant particle source from ionisation of the neutrals penetrated from the edge. The difference is more illustrative for the case of the variable edge cell used in the solver with $\rho$ (see figure 14). It is clear that the Simpson numerical integration on the sparse grid is not accurate for an exponentially decayed edge source, meanwhile it is satisfactory for the core source with a rather wide distribution. This could affect the difference in the simulations described above. 
To evaluate the effect of edge grid distribution in more detail, the edge source, which is most sensitive to the resolution, is arbitrarily removed in the simulation. Without the edge source, the difference between the solvers using $\rho$ and $\rho_{N}$ almost disappears. Another exercise of the simulation by removing all the sources, both from the edge and the core, exhibits almost no difference between the two solvers.

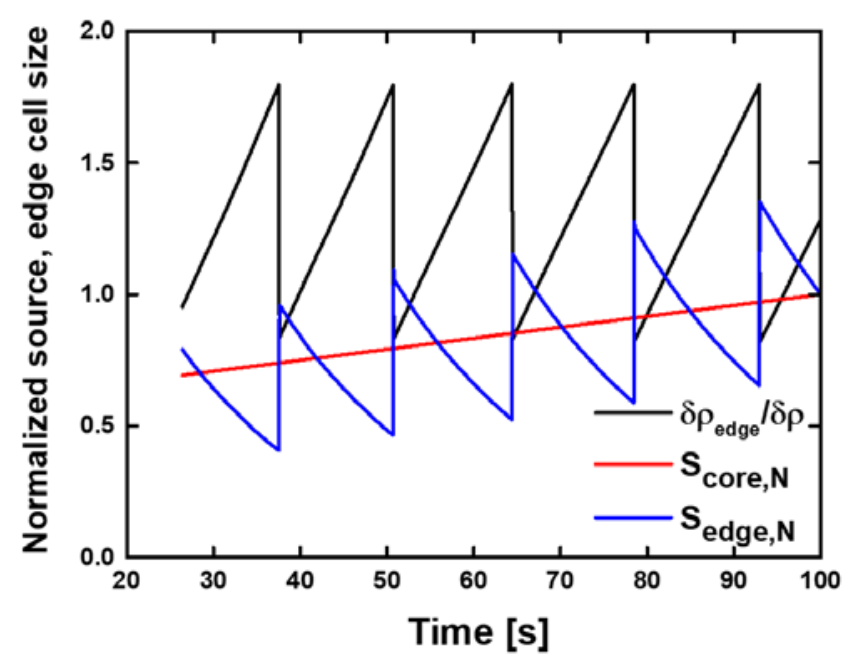

Figure 14. Evolution of the edge cell (black), ionisation source from the pellet (red), and ionisation source from the neutrals penetrated from the edge (blue) for a regular grid size $\Delta \rho / \rho \sim 1 / 30$ for the source distributions recommended for benchmarking.

\section{Volume evolution term}

As mentioned in the previous section, the transport solvers usually take $\rho$ or $\rho_{N}$ for the radial coordinate. In the case of using $\rho_{N}$, the so-called volume evolution term appears in the transport equation; $-\frac{1}{V^{\prime}} \frac{1}{2 \Phi_{a}} \frac{d \Phi_{a}}{d t} \frac{\partial}{\partial \rho_{N}} \rho_{N}\left(V^{\prime} n_{k}\right)$. This term is the correction of the virtual convection due to the expanding grid based on $\rho_{N}$ during the plasma expansion. Note that similar convective term, $-\frac{1}{V^{\prime}} \frac{1}{2 \Phi_{a}} \frac{d \Phi_{a}}{d t} \frac{\partial}{\partial \rho} \rho\left(V^{\prime} n_{k}\right)$, should be introduced in the ASTRA v.6 simulations, but just at the edge cell. It has a noticeable impact on the transport simulations only in the case when $\rho_{N}\left(\ln \left(\Phi_{\mathrm{a}}\right)\right) / d t$ is comparable with the convection term, $\left\langle\left(\nabla \rho_{N}\right)^{2}\right\rangle_{\mathrm{Vk}}$. 
The effects of the volume evolution term on the density evolution are investigated by comparing simulations with and without the volume evolution term using ASTRA v.7. The volume evolution term, the diffusion term, and the source term in equation (1) are arbitrarily adjusted to be comparable with each other to amplify the effect of the volume evolution term. Here we set the convection term to be zero for simplicity and the plasma volume expands as described previously but faster for full expansion up to $15 \mathrm{~s}$ to enhance the role of the volume evolution term in the ITER baseline scenario.
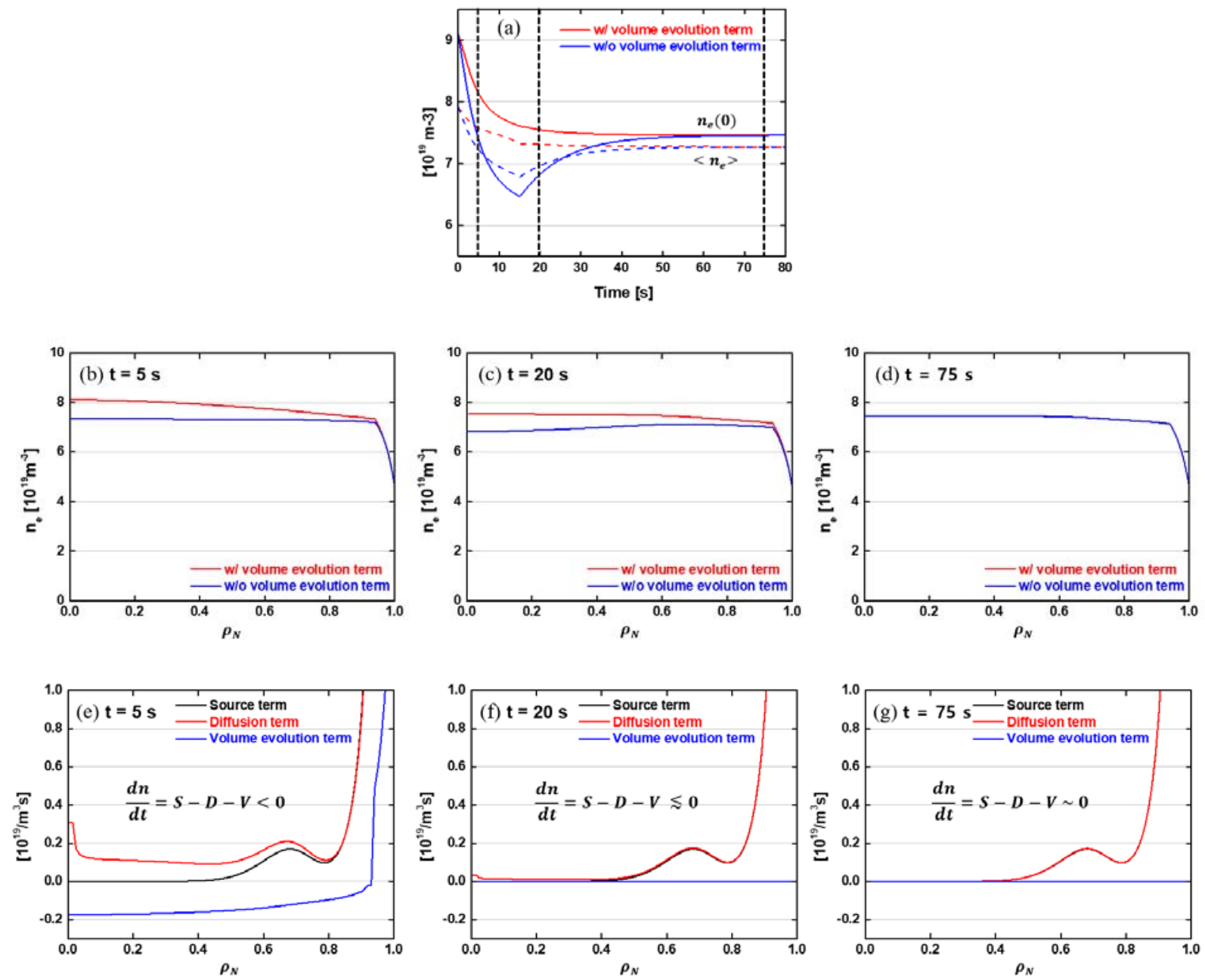

Figure 15. Time evolution of central electron density, $n_{e}(0)$ and volume-averaged electron density, $<n_{e}>(a)$, electron density profile for the vertical dashed lines in (a) at $5 s(b), 20 s(c)$ and $75 s(d)$ of the simulations with and without the volume evolution term in ASTRA v. 7 for the 
case with the plasma volume expansion up to $15 \mathrm{~s}$. The profiles of the source, diffusion, and volume evolution term at $5 \mathrm{~s}(\mathrm{e}), 20 \mathrm{~s}(\mathrm{f})$, and $75 \mathrm{~s}(\mathrm{~g})$ from the simulation with the volume evolution term. Here the plasma equilibrium is prescribed in the entire simulation.

The time evolution of the electron density is presented in figure 15 (a). As shown, solving with the volume evolution term exhibits the higher electron density during the plasma expansion phase, 0 to $15 \mathrm{~s}$. A transient behaviour is observed right after the plasma expansion for the simulation excluding the volume evolution term, whereas a smooth evolution is observed for the simulation including the volume evolution term but this difference between the two simulations becomes mitigated and almost disappears after the full expansion around $60 \mathrm{~s}$. The difference between the simulations result from the virtual inward convection effect due to the grid expansion as shown in figure 15 (d)-(f) which is strong in the core region. This virtual convection effect causes an increase of the core density compared with the case without the volume evolution term (see figure 15 (b)). For slowly expanding plasma, the effect of the volume evolution term becomes

smaller as $\frac{d \Phi_{a}}{d t}$ decreases, thus the difference between the simulations with and without the volume evolution term reduces.

As the simulations shown above are performed with prescribed time-evolving equilibria, the result can change when solving current diffusion, self-consistently. The difference in the density due to the volume evolution term affects the bootstrap current and temperature, affecting the current and pressure profiles and so the difference in the evolution of the equilibrium between the two cases, which in turn affects the difference in the density evolution. The results are shown in figure 16. 

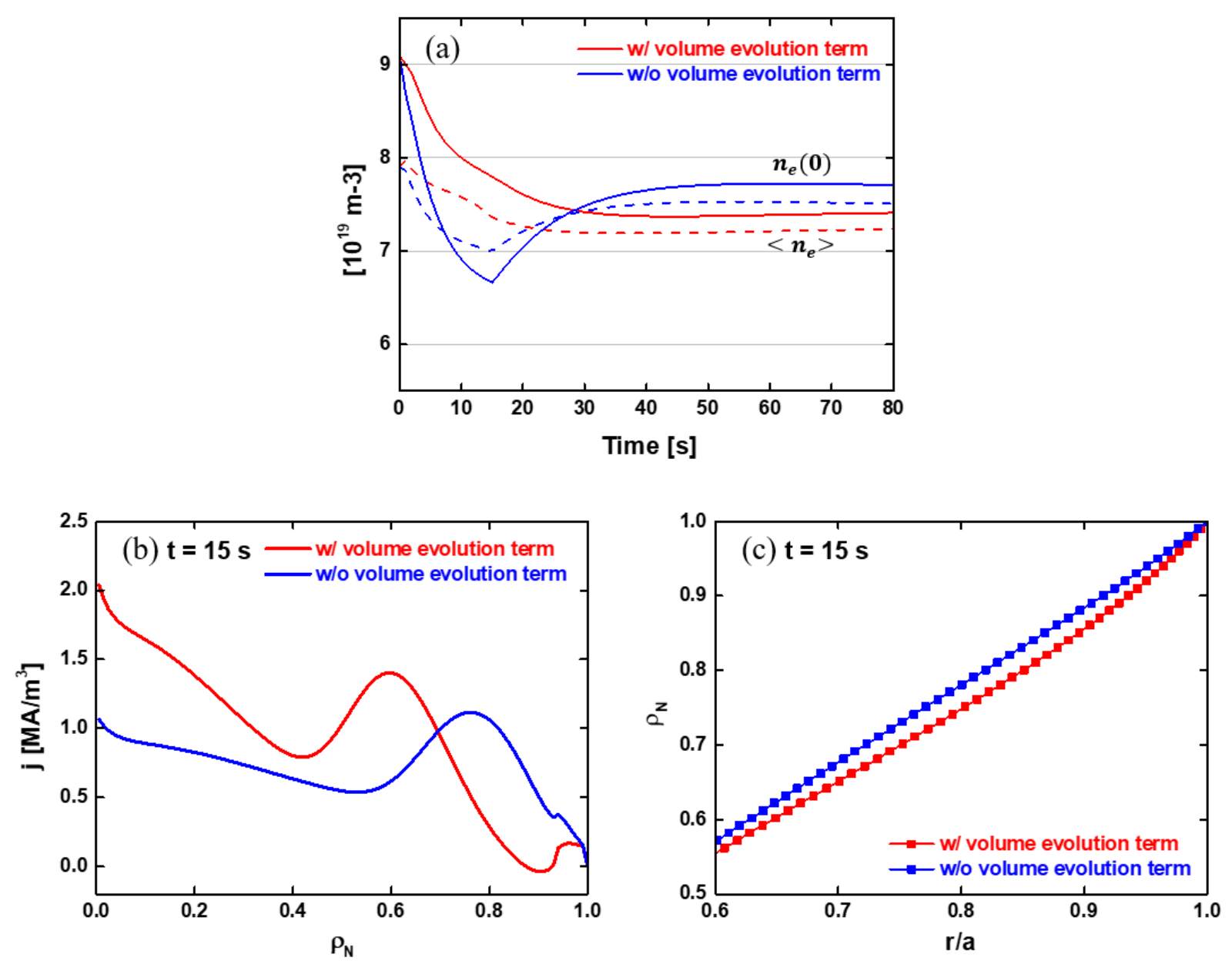

Figure 16. Time evolution of central electron density, $n_{e}(0)$ (blue lines), and volume-averaged electron density, $\left\langle n_{e}\right\rangle$ (red lines) (a), current density profile at $15 \mathrm{~s}$ (b), and the corresponding equilibrium difference (c) of the simulations with and without the volume evolution term in ASTRA v.7 for the case with the plasma volume expansion up to $15 \mathrm{~s}$. Here the current diffusion is solved.

As shown in figure 16 (a), for the case without the volume evolution term the density drops much faster at plasma expansion and starts to increase just after the end of expansion $(t=15 \mathrm{~s})$ in contrast to the case with the volume evolution term. It is also observed the lower density during the plasma expansion and the transient behaviour right after the expansion for the simulation without the volume evolution term while the current diffusion is solved. The core current as well as the electron density are reduced in the simulation excluding the volume evolution term as shown in figure 16 
(b). The trend is reversed at the edge to match the total current, so the coordinates based on the equilibrium differ in the real geometry between the two cases. In the simulation without the volume evolution term, the arrangement of $\rho_{N}$ becomes sparser in the edge region and denser in the core region (see figure 16 (c)), so the edge source increases whereas the core source is reduced because the source profile is given in $\rho_{N}$. Since the amount of the edge source is prescribed to be much larger than that of the core source, the density in the simulation without the volume evolution term becomes higher than that including the volume evolution term after the expansion.

Comparing with the simulation without solving the current diffusion, the density without the volume evolution term is higher as well due to the higher density source from the equilibrium difference. Since the current diffusion is much slower than the volume evolution, it takes a much longer time until the density in the two cases converges each other than the case without solving the current diffusion.

\subsection{Impact of the integrated plasma performance on the particle transport}

It is noteworthy that plasma parameters affecting the particle transport cannot be prescribed arbitrarily and controlled fully independently. In particular, boundary conditions and gas penetration through separatrix to the core depend on the fluxes of particles and power to the SOL $[3,21]$. Core fuelling is integrated with ELM pacing, divertor detachment, and ion cyclotron heating ( $\mathrm{ICH})$ coupling control as well as gas pumping [4]. Pellet penetration depends on the pedestal parameters which could be determined by the peeling-ballooning limit [4]. Here we discuss the interplay of plasma parameters affecting the particle transport and the results of fully integrated particle transport simulations. We also address the possibility of independent density control by pellet fuelling and gas puffing, and replacement of the discrete pellet fuelling by the socalled continuous pellet approximation for various pellet sizes to assess the impact of simplifications of the particle transport modelling on the accuracy of density predictions for ITER baseline scenario. These studies are done with ASTRA v.7.

For the sensitivity studies, we employ the results of SOLPS simulations [21] to obtain more realistic boundary conditions for the density and the temperature and the relation between the particle source and sink. The SOLPS simulations [7] have shown that the boundary density and the 
temperature increase with transition from L- to $\mathrm{H}$ - mode and the increase of the power loss to the SOL when a constant degree of divertor detachment is assumed. Thus, the opacity of the SOL to penetration of the gas from the edge noticeably increases. In the range of parameters of ITER plasmas, the edge fuelling in the H-mode operation becomes inefficient [7] and the core fuelling by the HFS pellet injection is required, meanwhile in the L-mode operation gas penetrated from the edge can remain the dominant fuelling source [4] as discussed in section 3.4. In these sensitivity studies, we use the dependencies of the boundary conditions, $\mathrm{T}_{\mathrm{i}, \mathrm{a}}, \mathrm{T}_{\mathrm{e}, \mathrm{a}}, \mathrm{n}_{\mathrm{e}, \mathrm{a}}, \mathrm{n}_{\mathrm{i}, \mathrm{a}}, \mathrm{n}_{\mathrm{He}, \mathrm{a}}$ and the source from the edge on the particle and power fluxes to SOL, $\mathrm{G}_{\text {sol }}, \mathrm{P}_{\text {sol }}$ and alpha heating, $\mathrm{P}_{\alpha}$, based on the SOLPS simulations [21] and the relation between the particle sources and sinks discussed in [4]. The results of SOLPS parameterisation for L- and H-mode operation of the ITER baseline scenario are displayed in figure 17.
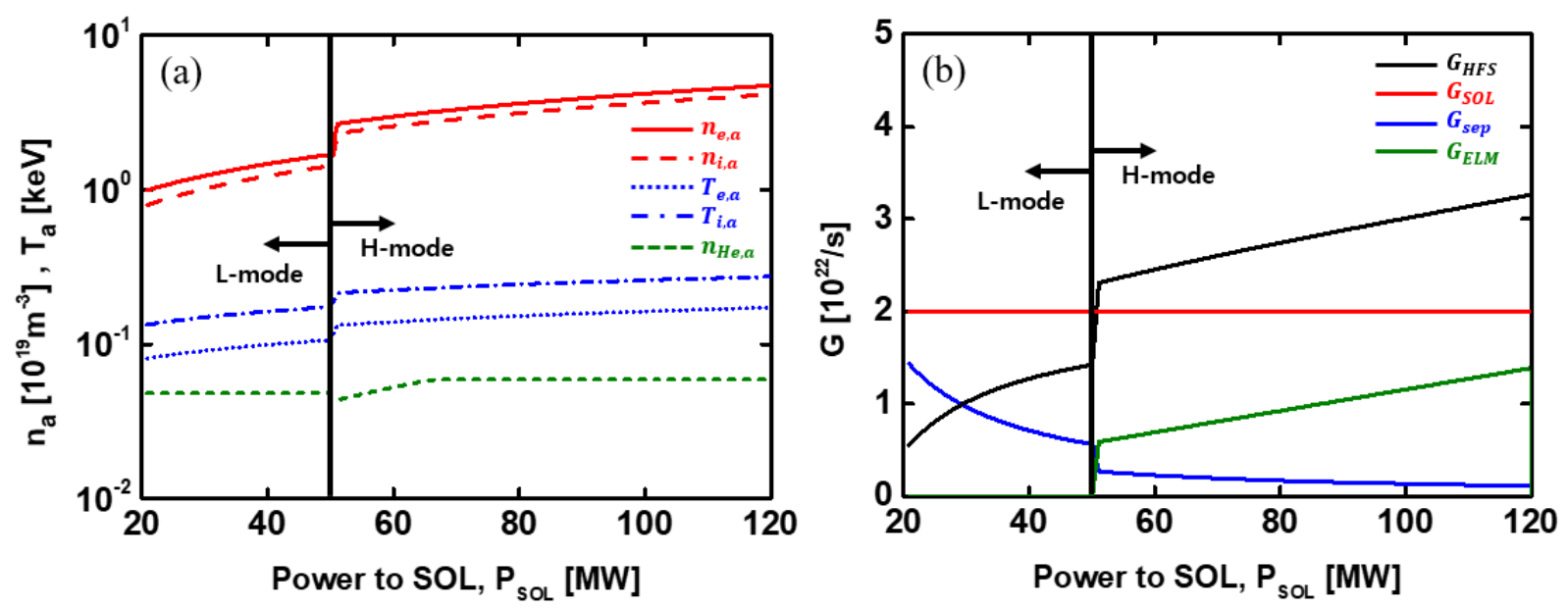

Figure 17. Plasma parameters derived from SOLPS parameterisation for ITER baseline scenario with pellet ELM pacing, $f_{p e l}=0.2 P_{\text {sol }} \Delta W_{E L M}, \triangle W_{E L M}=0.6 \mathrm{MJ}$, fuelling, and ELM pacing by pellets with $V_{p}=33 \mathrm{~mm}^{3}$ and $V_{p e l}=300 \mathrm{~m} / \mathrm{s}$, divertor detachment control with normalised divertor pressure, $\mu=0.7$, divertor pumping speed, $S_{\text {eng }}=57 \mathrm{~m}^{3} / \mathrm{s}$, Neon fraction $n_{N e s} / n_{e s}=1 \%$, where $\mu=0.67 p_{n} / P_{S O L}^{0.39}(P a, M W)=1$ [21], where $p_{n}$ is the modelled neutral pressure and PSOL is the edge power flow. The divertor neutral pressure can be controlled by pumping $\left(S_{\text {eng }}\right)$ [21]; for a given fuelling rate $G_{D T}$, $p_{n}=4.79\left(G_{D T} / S_{\text {eng }}\right)^{0.83} / P_{S O L}^{0.13}$ : (a) boundary conditions; electron and ion boundary temperatures, $T_{e, a}, T_{i, a}$, electron, fuel and He boundary 
densities, $n_{e, a}, n_{i, a}, n_{H e, a}$, (b) particle sinks and sources; particle loss to SOL with diffusion and convection, $G_{\text {sol, }}$ particle loss with ELMs, $G_{E L M}$, particle source from the HFS pellet injection, GHFS, ionisation source from the gas penetrated from the edge, $G_{s e p}$.

Note that in the low power L-mode operation, the ionisation source due to the gas, penetrated from the edge provides $30-70 \%$ of total core fuelling, whereas for the H-mode operation it becomes negligible in comparison with the pellet fuelling. Thus, the accuracy of the ionisation source from the edge is important only at the Ohmic and L-mode phases, meanwhile the accuracy of the pellet modelling is important for the H-mode phases. It is noteworthy that for the H-mode operation the fraction of the particle loss with ELMs (GELM) becomes comparable with the diffusive and convective loss $\left(\mathrm{G}_{\mathrm{sol}}\right)$ (figure 17 (b)). In the simulations, we assumed fully convective ELMs with particle loss per ELM, $\Delta \mathrm{N}_{\text {pel }}=\mathrm{n}_{\text {e,ped }} \mathrm{V}_{\text {plasm }} \Delta \mathrm{W}_{\mathrm{ELM}} / \mathrm{W}_{\text {ped }}$ [4], where $\mathrm{n}_{\mathrm{e}, \text { ped }}$ is the pedestal density, $\mathrm{V}_{\text {plasm }}$ is the plasma volume, $\Delta \mathrm{W}_{\text {ELM }}$ is the energy loss per ELM, and $\mathrm{W}_{\text {ped }}$ is the pedestal energy content.

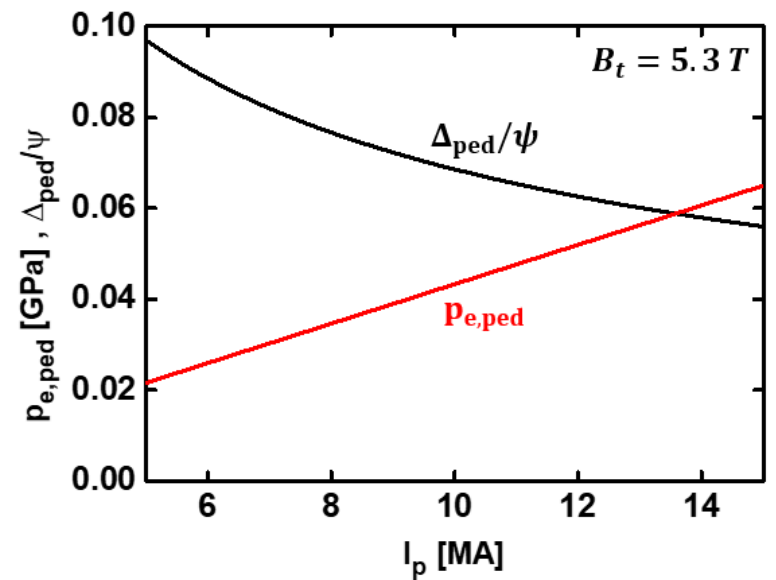

Figure 18. Relative pedestal width and pressure of the electrons at the top of pedestal predicted by EPED1+SOLPS scaling for the ITER baseline scenario

It was found that the efficiency of the fuelling by pellets decreases with the decrease of the pellet particle deposition depth [36] and increases with the number of particles penetrated through the pedestal to the core. The depth of pellet penetration reduces with the increase of the electron temperature and density at the pedestal and with decrease of the pellet size, $\mathrm{V}_{\mathrm{p}}$ and the pellet speed, 
$\mathrm{V}_{\text {pel }}$ [22]. The pedestal width, density, and temperature are chosen from the peeling-ballooning limit, which can be determined by the EPED1+SOLPS scaling [4]. According to [4] the pedestal height increases with the plasma current and magnetic field, $\mathrm{p}_{\text {eped }} \sim \mathrm{nT}_{\mathrm{ped}} \sim \mathrm{I}_{\mathrm{p}} \mathrm{B}$, and pedestal width, $\Delta_{\mathrm{xped}} \sim\left(\mathrm{B} / \mathrm{I}_{\mathrm{p}}\right)^{0.5}$. Thus, in our simulations, we consider the full-field ITER operation, $\mathrm{B}=5.3 \mathrm{~T}$, as the most challenging for pellet fuelling with the pedestal height and width derived from the scaling (figure 18).

Another typical simplification is the treatment of pellet fuelling in the continuous pellet approximation, where the discrete pellets of the size $V_{p, H F S}$ and the frequency fHFS are replaced by the continuous source $\mathrm{G}_{\mathrm{HFS}}=\mathrm{n}_{\mathrm{pH}} \times \mathrm{V}_{\mathrm{p}, \mathrm{HFS}} \times \mathrm{f}_{\mathrm{HFS}}$, where $\mathrm{n}_{\mathrm{pH}}=6 \times 10^{28} \mathrm{~m}^{-3}$ is the density of the hydrogen ice. To clarify the impact of this assumption on the accuracy of the pellet fuelling efficiency in the sensitivity studies, we simulate only the particle transport with a single ion species and a prescribed temperature profile. In our simulations, we assumed that temperature profiles are prescribed analytically as functions of $\rho_{\mathrm{N}}$ with a linear decrease in the pedestal region from pedestal top values, $T_{\text {eped }} \sim 1 / \mathrm{n}_{\text {eped }}$ to separatrix values, $T_{\text {ea, }}$, determined from the SOLPS parameterisation. The pellet injection geometry was chosen with the pellet speed of $V_{\text {pel }}=300 \mathrm{~m} / \mathrm{s}$ and the pellet sizes of $\mathrm{V}_{\mathrm{p}}=33,50,90 \mathrm{~mm}^{3}$. We used the electron particle solver with the same transport coefficients used above in the benchmarking of the particle transport solvers, $\mathrm{D}=\mathrm{D}_{\text {bench, }} \mathrm{v}=\mathrm{v}$ bench, for the $\mathrm{H}$-mode and $\mathrm{D}=0.5 \mathrm{D}$ bench, $\mathrm{v}=0.5 \mathrm{v}$ bench, for the L-mode simulations.

The sensitivity studies of fuelling efficiency by HFS pellets to the pellet size are carried out for the L- and H-mode with continuous and discrete pellet approximations by ASTRA with SMART. Boundary conditions are calculated consistently from SOLPS parameterisation and the pedestal parameters were fitted to EPED1+SOLPS predictions. The results are presented in figures 19 and 20. 


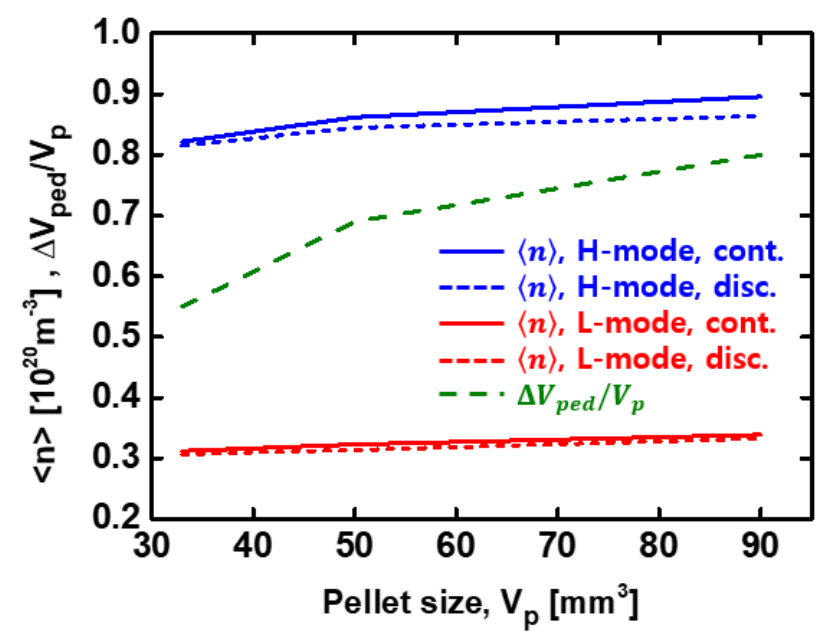

Figure 19. Density predictions for L-and H-mode and pellet fraction penetrated through the pedestal $\left(\Delta V_{p e d} / V_{p}\right)$ in continuous and discrete pellet approximations for the ITER baseline scenario.

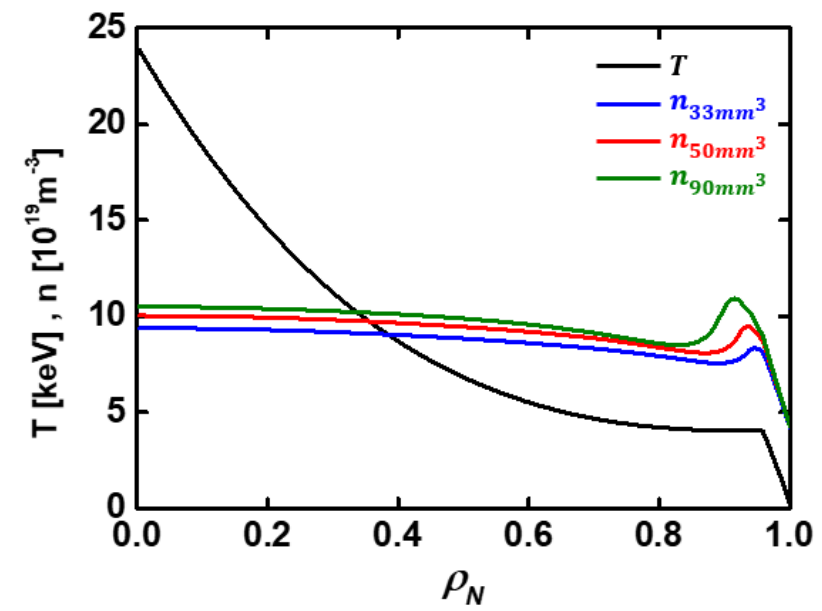

Figure 20. Radial profiles in $\rho_{N}$ for the target plasma temperature and post-pellet density for different pellet sizes, 33, 50, $90 \mathrm{~mm}^{3}$ for the ITER baseline scenario.

According to the simulation for the same HFS pellet fuelling, the density is predicted to increase by $\sim 10 \%$ with the increase of the pellet size from 33 to $90 \mathrm{~mm}^{3}$, which is less than the difference in the pellet fraction penetrated though the pedestal $\left(\Delta V_{\text {ped }} / V_{p}\right)$ (figure 19). This could be caused by the efficient reduction of transport in the edge transport barrier (ETB). The difference of 
predictions for continuous and discrete approximations in the L-mode is small due to the high fraction of the fuelling from the gas penetrated from the edge. For the H-mode the difference increases with the pellet size up to $\sim 7 \%$ for $90 \mathrm{~mm}^{3}$ pellets. Figure 20 demonstrates how the difference of the details of fuelling at the outer $20 \%$ of minor radius discussed above for gas and pellet fuelling can affect the core density predictions. In the case considered, the difference of the central density predictions is about $10 \%$. Such a difference can be more pronounced in integrated modelling predicting the fusion power as $\mathrm{P}_{\text {fusion }} \sim \mathrm{n}^{2}$. On the other hand, because the central source of He ash also increases together with the sink of the fuel in the DT reaction with increasing fusion power, the final impact of a core density increase on the fusion power at the axis will be weaker than $\mathrm{n}^{2}$.

Note that it is possible to expect noticeable difference between predictions of the continuous and discrete pellet models, when $\mathrm{D}$ and $\mathrm{T}$ transport is simulated separately because of the mutual impact of the $\mathrm{D}$ and $\mathrm{T}$ profiles on the fuel transport and appearance of reversed gradients in the pellet affected region, which has an impact on the first principal model predictions [43, 44].

\section{Conclusions}

The particle transport codes are benchmarked with the integrated transport codes used for ITER scenario modelling within ITPA IOS Topical Group. To make possible the benchmarking we identified the differences in the transport equation between particle transport solvers and then unified the definitions. It was found that some of the solvers assume different metric coefficients for the diffusive and the convective terms. For such solvers the ratio $\mathrm{D} / \mathrm{v}$ cannot be directly

translated to the density profile peaking, $\frac{R}{L_{n}}=-R n^{\prime} / n$, which makes less trivial the comparison with the experiments. On the other hand, the selection of metric coefficients made with these solvers is the only one for which v corresponds to the flux surface average of the orthogonal local fluxes and for which transport coefficients $\mathrm{D}$ and $\mathrm{v}$ are invariant with respect to the choice of the flux surface label as detailed in [11]. At the first phase, we compared the particle transport solvers for the ions and the electrons with prescribed transport coefficients, stationary sources, and boundary conditions for plasma parameters, expected in a stationary phase of the ITER baseline ELMy H-mode scenario. Firstly, we checked and confirmed the particle flow balance in each 
particle transport solvers. Secondly, we benchmarked the ion transport solvers as step 1 . Then the ion solvers were adopted for emulation of the electron transport solvers by modifying the pinch term to be benchmarked with the electron solvers as step 2 . The calculated density agrees within $3 \%$ in both steps. However, differences of the particle profile predictions are observed between the two steps where the same particle diffusivity and pinch velocity are applied to each species. Such a big difference is caused by the quasi-neutrality condition; the ion solvers predict the ion density and the electron density is calculated through quasi-neutrality and the electron solvers vice versa. The presence of the noticeable fraction of helium ash with relatively high peaking can increase the difference between the fuel and the electron density gradients. For present day machines $\mathrm{n}_{\mathrm{i}}{ }^{\prime} \sim \mathrm{n}_{\mathrm{e}}{ }^{\prime}$, and the difference vanishes to zero. Thus, such a big difference is the specific feature of the burning plasmas. Therefore, for fusion reactors it is extremely important to use appropriate theory-based transport models to choose the adequate solvers for the electron and the ion transport. For empirical and semi-empirical particle transport models, the predictive capability becomes more uncertain. Note that the electron density peaking affects the temperature peaking for some transport models and thus, the fusion power, the fuel ion peaking affects the fusion power directly by Pfusion $\operatorname{nDnT}(\rho=0)$. We also benchmarked the pellet fuelling modules in the stationary phase. Two pellet fuelling models were benchmarked for the prescribed target plasma and pellet injection parameters to reveal the sensitivity of the deposition profiles to the injection side, the pellet volume, the pedestal parameters, and the separatrix parameters. Modelling of the HFS fuelling demonstrates noticeable dependence of the depth of the particle source on the injected pellet size and weak sensitivity to the other parameters for both models. For LFS, small residual fuelling is predicted. As the amount of residual fuelling can affect the integrated control of fuelling, ELM mitigation, and divertor detachment, the numerical analysis of such possible impact and dedicated validation of pellet modelling of the LFS pellets is required. Note that for reactor simulations all codes predict pretty shallow fuelling, $\rho / \rho_{a}>0.8$, both for the gas and the pellet fuelling. Therefore, it is difficult to expect strong impact on the central fuel density and so on the fusion power. Meanwhile the difference in the fuelling predictions still can affect the pedestal stability, like in present day machines. The edge gas fuelling was also benchmarked in the stationary phase. Three codes were employed for the benchmark for the prescribed plasma conditions to investigate sensitivities of the source profile predictions with respect to the background plasma conditions and detailed model assumptions. The main differences between code predictions is found to result from assumptions 
of geometry, recycling, neutral energy distribution, and reaction cross-sections. It may be worth pointing out that in present day machines a particularly accurate description of cold neutral sources may be required for specific interpretative modelling tasks related to edge transport and stability. In particular, the prediction of core neutral sources at high precision may be essential for the analysis of the effective edge, pedestal transport coefficients [27, 37], and of the effect of edge profile shape variations (e.g. a shift in temperature vs. density barrier locations [38]) on the MHD stability $[39,40]$. In future tokamaks like ITER and DEMO, the SOL becomes opaque for penetration of the neutrals from the edge, thus the details of the edge gas penetration are less important.

At the second phase of benchmarking, we compared the particle transport in the timeevolving scenario from the L-mode ramp-up phase, L-H transition, flattop H-mode phase, H-L transition, and L-mode ramp-down phase. The ion and the electron solvers are benchmarked together with prescribed evolutions of the plasma configuration, particle transport coefficients, sources, and boundary conditions. The code predictions are within $6 \%$ and the differences are mainly originated from the pedestal region.

To reveal the origins of the differences observed between codes during the benchmarking, we evaluate the effect of the equilibrium, the grid resolution, the radial coordinate used in the solver ( $\rho$ or $\rho_{N}$ ) and the grid distribution, and the volume evolution term. Firstly, the difference in equilibrium can affect the total integrated particle source as well as the number of particles in the plasma volume. Secondly, the number of grid can influence the fuelling rate, the number of the $\mathrm{He}$ particle, and in particular the location of the pedestal which ends up with the different density profiles. Thirdly, the choice of the radial coordinate, $\rho$ or $\rho_{N}$ is found to be able to significantly alter the result as the integrated source and the diffusive flux become different when the plasma edge source is dominant and the radial grid is sparse. The main difference is originated from the edge region where the source is large and the gradient is sharp due to the pedestal. But increase of the number of the grid nodes increases the accuracy of numerical approximation of the edge source making the difference in the predicted density smaller. Lastly, it was revealed that the volume evolution term can be dominant when the plasma shape varies faster than the transport time scale $\left(\tau_{\text {shaping }} \gg \tau_{\text {particle }}\right)$ but not significant vice versa. 


\section{Acknowledgment}

Disclaimer: The views and opinions expressed herein do not necessarily reflect those of the ITER Organisation nor those of the European Commission. Authors would like to express our gratitude to Dr. Emiliano Fable for fruitful discussion on ASTRA v.7 and The Research Institute of Energy and Resources, Seoul National University. This work was supported by the Ministry of Science, ICT and Future Planning of the Republic of Korea under the Korean ITER project contract and the U.S. Department of Energy, Office of Science, Office of Fusion Energy Sciences, under contract DE-AC02-09CH1 1466 and in part by the Swiss National Science Foundation. This work has been carried out within the framework of the EUROfusion Consortium and has received funding from the Euratom research and training programme 2014-2018 under grant agreement No 633053 and from the RCUK Energy Programme [grant number EP/P012450/1].

\section{References}

[1] A.C.C. Sips, et al., Phys. Plasmas 22021804 (2015)

[2] A. Loarte, Nucl. Fusion 54123014 (2014)

[3] M. Romanelli, et al., Nucl. Fusion 55093008 (2015)

[4] A.R. Polevoi, et al., Nucl Fusion 57022014 (2017)

[5] L. Garzotti et al., Nucl. Fusion 52013002 (2012)

[6] A. Loarte, et al., Nucl. Fusion 53083031 (2013)

[7] A.S. Kukushkin, et al., J. Nucl. Mater. 415 S497 (2011)

[8] J. Garcia, et al., Nucl. Fusion 54093010 (2014)

[9] L. Garzotti, et al., Nucl. Fusion 46994 (2006)

[10] C.E. Kessel, et al., Nucl. Fusion 471274 (2007)

[11] G. Pereverzev, et al., IPP-Report IPP 5/98 (2002)

[12] E. Fable, et al., Plasma Phys. Control. Fusion 55124028 (2013)

[13] D. Kalupin, et al., Nucl. Fusion 53123007 (2013)

[14] F. Poli, J. Sachdev, J. Breslau, M. Gorelenkova, X. Yuan, (2018, June 27), TRANSP, [Computer software], doi:10.11578/dc.20180627.4.

[15] F. Felici, et al., Nucl. Fusion 58096006 (2018)

[16] H. Shirai, et al., Plasma Phys. Control. Fusion 421193 (2000) 
[17] J. F. Artaud, et al., Nucl. Fusion 50, 043001 (2010)

[18] J.M. Park, et al, Comput. Phys. Commun. 214, 1 (2017)

[19] M. Romanelli, et al., Plasma Fusion Res. 93403023 (2014)

[20] M. Honda, et al., Nucl. Fusion 46580 (2006)

[21] H.D. Pacher, et al., J. Nucl. Mater. 463591 (2015)

[22] A.R Polevoi, M. Shimada, Plasma Phys. Control. Fusion 431525 (2001)

[23] B. Pégourié, et al., Nucl. Fusion 4744 (2007)

[24] B. Pégourié, et al., Plasma Phys. Contr. Fusion 51124023 (2009)

[25] M. Valovic, et al., Plasma Phys. Contr. Fusion 461877 (2004)

[26] F. Imbeaux, et al., Nucl. Fusion 51083026 (2011)

[27] F. Koechl, et al., Nucl. Fusion 57086023 (2017)

[28] A.R. Polevoi, et al., Nucl. Fusion 55063019 (2015)

[29] S. Tamor, J. Comput. Phys. 40104 (1981)

[30] D. Reiter, J. Nucl. Mater. 19680 (1992)

[31] R.L. Freeman, E.M. Jones, Report CLM-R 137461 Culham Laboratory (1974)

[32] V.A. Abramov, V.V. Vikhrev, O.P.Pogutse, Fizika Plazmy 3 N3 512 (1977)

[33] R.K. Janev, W.D. Langer, K.J. Evans, D.E.J. Post, "Elementary Processes in HydrogenHelium Plasmas", Springer Verlag Berlin Heidelberg (1987)

[34] M.H. Hughes, D.E. Post, J. Comput. Phys. 2843 (1978)

[35] S. Wiesen, et al., J. Nucl. Mater. 463480 (2015)

[36] M. Valovic, et al., Nucl. Fusion 48075006 (2008)

[37] M. Kotschenreuther, et al., "Gyrokinetic Analysis and Simulations of Pedestals", Preprint: 2018 IAEA Fusion Energy Conference, Gandhinagar [TH/2-2]

[38] F. Koechl, et al., Plasma Phys. Contr. Fusion 60074008 (2018)

[39] J.S. Lönnroth, et al., Nucl. Fusion 51013003 (2011)

[40] E. Stefanikova, et al., Nucl. Fusion 58056010 (2018)

[41] G. Kocsis, et al., Nucl. Fusion 471166 (2007)

[42] M. Shimada, et al., "Progress in the ITER Physics Basis Chapter 1: Overview and summary”, 2007 Nucl. Fusion 47 S1

[43] A. Loarte, et al., 2013 Nucl. Fusion 53083031 (2013)

[44] B. Baiocchi, et al, Nucl. Fusion 55123001 (2015) 


\section{Appendix}

\section{Setup for simulations in stationary phase}

- Edge particle source:

$\mathrm{S}(\Phi)=\mathrm{S}_{0} \exp \left[15\left(\Phi-\Phi_{\mathrm{a}}\right) / \Phi_{\mathrm{a}}\right]$, where $\mathrm{S}_{0}=7.5 \times 10^{20}$ atoms $/ \mathrm{m}^{3} / \mathrm{s}$.

$\mathrm{S}_{\mathrm{D}}(\Phi)=\mathrm{S}_{\mathrm{T}}(\Phi)=0.5 \times \mathrm{S}(\Phi)$ for ion solvers

- Core particle source by pellet fuelling (continuous):

$\mathrm{S}(\Phi)=\mathrm{C} \times \mathrm{d}^{2} \times\left(\Phi / \Phi_{\mathrm{a}}\right)^{6.5} \times\left[1-\left(\Phi / \Phi_{\mathrm{a}}\right)\right]^{8.5} /\left\{\mathrm{d}^{2}+\left[\left(\Phi / \Phi_{\mathrm{a}}\right)-0.5\right]^{2}\right\}$

, where $\mathrm{C}=0.25 \times 10^{24}$ and $\mathrm{d}=0.225$.

$\mathrm{S}_{\mathrm{D}}(\Phi)=\mathrm{S}_{\mathrm{T}}(\Phi)=0.5 \times \mathrm{S}(\Phi)$ for ion solvers

- Particle diffusivity:

$\mathrm{D}(\Phi)=\mathrm{D}_{0}+\mathrm{D}_{1}\left(\Phi / \Phi_{\mathrm{a}}\right)^{2}$ for $\Phi<\Phi_{\text {ped }}$

$\mathrm{D}(\Phi)=\mathrm{D}_{2}$ for $\Phi \geq \Phi_{\text {ped }}$

, where $\mathrm{D}_{0}=0.5 \mathrm{~m}^{2} / \mathrm{s}, \mathrm{D}_{1}=1.0 \mathrm{~m}^{2} / \mathrm{s}, \mathrm{D}_{2}=0.11 \mathrm{~m}^{2} / \mathrm{s}, \Phi_{\text {ped }}$ is $\Phi$ at the pedestal top, $\Phi_{\text {ped }} / \Phi_{\mathrm{a}}=0.88$.

- Pinch number:

$\mathrm{R}_{0} \times \mathrm{V}_{0} / \mathrm{D}=\mathrm{V}_{0} \times\left(\Phi / \Phi_{\mathrm{a}}\right)^{1 / 2}$, where $\mathrm{R}_{0}=6.2 \mathrm{~m}, \mathrm{~V}_{0}=1.385$ and positive sign corresponds the inward pinch.

$\mathrm{v}(\Phi)=\mathrm{v}_{0}$ for transport solvers with the definition of flux,

$\Gamma=-D \frac{\partial n}{\partial \rho}\left\langle(\nabla \rho)^{2}\right\rangle+n v\left\langle(\nabla \rho)^{2}\right\rangle$

$\mathrm{v}(\Phi)=-\mathrm{v}_{0}$ for transport solvers with the definition of flux,

$\Gamma=-D \frac{\partial n}{\partial \rho}\left\langle(\nabla \rho)^{2}\right\rangle-n v\left\langle(\nabla \rho)^{2}\right\rangle$ 
$\mathrm{v}(\Phi)=\mathrm{v}_{0}\left\langle(\nabla \rho)^{2}\right\rangle /\langle|\nabla \rho|\rangle$ for transport solvers with the definition of flux,

$\Gamma=-D \frac{\partial n}{\partial \rho}\left\langle(\nabla \rho)^{2}\right\rangle+n v\langle|\nabla \rho|\rangle$

$\mathrm{v}(\Phi)=-\mathrm{v} 0\left\langle(\nabla \rho)^{2}\right\rangle /\langle|\nabla \rho|\rangle$ for transport solvers with the definition of flux,

$\Gamma=-D \frac{\partial n}{\partial \rho}\left\langle(\nabla \rho)^{2}\right\rangle-n v\langle|\nabla \rho|\rangle$

- The edge boundary condition:

$\mathrm{n}_{\mathrm{ea}}=4.6 \times 10^{19} / \mathrm{m}^{3}$, close to the predictions of the SOLPS simulations of the baseline scenario with $\mathrm{P}_{\mathrm{sol}}=100 \mathrm{MW}[15]$.

$\mathrm{n}_{\mathrm{Da}}=\mathrm{n}_{\mathrm{Ta}}=1.936 \times 10^{19} / \mathrm{m}^{3}$

$\mathrm{n}_{\text {Hea }}=0.0 \times 10^{19} / \mathrm{m}^{3}$

$\mathrm{n}_{\text {Bea }}=0.1636 \times 10^{19} / \mathrm{m}^{3}$

$\mathrm{n}_{\text {Ara }}=0.004093 \times 10^{19} / \mathrm{m}^{3}$

\section{Setup for time-varying simulations}

- Total discharge time $=710 \mathrm{~s}$ :

Ramp-up phase $=100 \mathrm{~s}(0-100 \mathrm{~s})$

Flattop phase $=450 \mathrm{~s}(100-550 \mathrm{~s})$

Ramp-down phase $=160 \mathrm{~s}(550-710 \mathrm{~s})$

- Scenario for plasma current:

\begin{tabular}{|l|l|l|l|l|l|l|l|l|l|l|l|l|l|l|l|l|l|l|}
\hline $\begin{array}{l}\text { Time } \\
(\mathrm{s})\end{array}$ & 1.5 & 2 & 3 & 10 & 20 & 30 & 40 & 50 & 75 & $\begin{array}{l}100- \\
550\end{array}$ & 570 & 590 & 610 & 630 & 650 & 670 & 690 & 710 \\
\hline $\mathrm{I}_{\mathrm{p}}$ & 0.5 & 0.735 & 1.20 & 4.50 & 6.5 & 8.16 & 9.44 & 10.6 & 13.5 & 15.0 & 13.5 & 12.0 & 10.5 & 9.0 & 7.5 & 6.0 & 4.5 & 3.0 \\
(MA) & & & & & & & & & & & & & & & & & & \\
\hline
\end{tabular}




\section{Ramp-up phase: $t=0-100 \mathrm{~s}$}

- Edge particle source for electrons:

$\mathrm{S}(\Phi)=\mathrm{S}_{0} \exp \left[10\left(\Phi-\Phi_{\mathrm{a}}\right) / \Phi_{\mathrm{a}}\right], \mathrm{S}_{0}=2.0 \times 10^{21}$ atom $/ \mathrm{m}^{3}$

- Electron particle diffusivity:

$\mathrm{D}_{\mathrm{e}}(\Phi)=\mathrm{D}_{0}+\mathrm{D}_{1}\left(\Phi / \Phi_{\mathrm{a}}\right)^{2}$ for $\Phi<0.88 \Phi_{\mathrm{a}}$

$\mathrm{D}_{\mathrm{e}}(\Phi)=\mathrm{D}_{2}$ for $\Phi \geq 0.88 \Phi_{\mathrm{a}}$

, where $\mathrm{D}_{0}=0.5 \mathrm{~m}^{2} / \mathrm{s}, \mathrm{D}_{1}=1.0 \mathrm{~m}^{2} / \mathrm{s}, \mathrm{D}_{2}=1.27 \mathrm{~m}^{2} / \mathrm{s}$

- Electron pinch number:

$\mathrm{R}_{0} \times \mathrm{V}_{0} / \mathrm{D}=\mathrm{V}_{0} \times\left(\Phi / \Phi_{\mathrm{a}}\right)^{1 / 2}$, where $\mathrm{R}_{0}=6.2 \mathrm{~m}, \mathrm{~V}_{0}=1.385$ and positive sign corresponds the inward pinch.

$\mathrm{v}(\Phi)=\mathrm{v}_{0}$ for transport solvers with the definition of flux,

$$
\Gamma=-D \frac{\partial n}{\partial \rho}\left\langle(\nabla \rho)^{2}\right\rangle+n v\left\langle(\nabla \rho)^{2}\right\rangle
$$

$\mathrm{v}(\Phi)=-\mathrm{v}_{0}$ for transport solvers with the definition of flux,

$$
\Gamma=-D \frac{\partial n}{\partial \rho}\left\langle(\nabla \rho)^{2}\right\rangle-n v\left\langle(\nabla \rho)^{2}\right\rangle
$$

$\mathrm{v}(\Phi)=\mathrm{v}_{0}\left\langle(\nabla \rho)^{2}\right\rangle /\langle|\nabla \rho|\rangle$ for transport solvers with the definition of flux,

$\Gamma=-D \frac{\partial n}{\partial \rho}\left\langle(\nabla \rho)^{2}\right\rangle+n v\langle|\nabla \rho|\rangle$

$\mathrm{v}(\Phi)=-\mathrm{v} 0\left\langle(\nabla \rho)^{2}\right\rangle /\langle|\nabla \rho|\rangle$ for transport solvers with the definition of flux,

$\Gamma=-D \frac{\partial n}{\partial \rho}\left\langle(\nabla \rho)^{2}\right\rangle-n v\langle|\nabla \rho|\rangle$

- Boundary condition: 


\begin{tabular}{|l|l|l|l|l|l|l|l|l|l|}
\hline Time $(\mathrm{s})$ & 0 & 5 & 10 & 20 & 30 & 40 & 50 & 75 & 100 \\
\hline $\begin{array}{l}\mathrm{n}_{\text {ea }} \\
\left(10^{19} / \mathrm{m}^{3}\right)\end{array}$ & 0.16 & 0.32 & 0.563 & 0.968 & 1.13 & 1.24 & 1.35 & 1.64 & 1.8 \\
\hline
\end{tabular}

- Impurity contents:

$\mathrm{n}_{\mathrm{Be}} / \mathrm{n}_{\mathrm{e}}=0.02, \mathrm{n}_{\mathrm{Ar}} / \mathrm{n}_{\mathrm{e}}=0.0$

\section{L-H and Flattop phase: $t=100-550 \mathrm{~s}$}

- Edge particle source for electrons:

$\mathrm{S}(\Phi)=\mathrm{S}_{0} \exp \left[15\left(\Phi-\Phi_{\mathrm{a}}\right) / \Phi_{\mathrm{a}}\right], \mathrm{S}_{0}=7.5 \times 10^{20}$ atom $/ \mathrm{m}^{3}$

- Core particle source for electron by pellet fuelling (continuous) during 125-550 s:

$\mathrm{S}(\Phi)=\mathrm{C} \times \mathrm{d}^{2} \times\left(\Phi / \Phi_{\mathrm{a}}\right)^{6.5} \times\left[1-\left(\Phi / \Phi_{\mathrm{a}}\right)\right]^{8.5} /\left\{\mathrm{d}^{2}+\left[\left(\Phi / \Phi_{\mathrm{a}}\right)-0.5\right]^{2}\right\}$

, where $\mathrm{C}=0.17325 \times 10^{24}, \mathrm{~d}=0.225$

- Electron particle diffusivity:

$\mathrm{D}_{\mathrm{e}}(\Phi)=\mathrm{D}_{0}+\mathrm{D}_{1}\left(\Phi / \Phi_{\mathrm{a}}\right)^{2}$ for $\Phi<\Phi_{\text {ped }}$

$\mathrm{D}_{\mathrm{e}}(\Phi)=\mathrm{D}_{2}$ for $\Phi \geq \Phi_{\text {ped }}$

, where $\mathrm{D}_{0}=0.5 \mathrm{~m}^{2} / \mathrm{s}, \mathrm{D}_{1}=1.0 \mathrm{~m}^{2} / \mathrm{s}, \mathrm{D}_{2}=0.1 \mathrm{~m}^{2} / \mathrm{s}$, and $\Phi_{\text {ped }}=0.88 \Phi_{\mathrm{a}}$

- Electron pinch number:

$\mathrm{R}_{0} \times \mathrm{V}_{0} / \mathrm{D}=\mathrm{V}_{0} \times\left(\Phi / \Phi_{\mathrm{a}}\right)^{1 / 2}$, where $\mathrm{R}_{0}=6.2 \mathrm{~m}, \mathrm{~V}_{0}=1.385$ and positive sign corresponds the inward pinch.

$\mathrm{v}(\Phi)=\mathrm{v}_{0}$ for transport solvers with the definition of flux,

$$
\Gamma=-D \frac{\partial n}{\partial \rho}\left\langle(\nabla \rho)^{2}\right\rangle+n v\left\langle(\nabla \rho)^{2}\right\rangle
$$

$\mathrm{v}(\Phi)=-\mathrm{v} 0$ for transport solvers with the definition of flux,

$$
\Gamma=-D \frac{\partial n}{\partial \rho}\left\langle(\nabla \rho)^{2}\right\rangle-n v\left\langle(\nabla \rho)^{2}\right\rangle
$$


$\mathrm{v}(\Phi)=\mathrm{v}_{0}\left\langle(\nabla \rho)^{2}\right\rangle /\langle|\nabla \rho|\rangle$ for transport solvers with the definition of flux,

$\Gamma=-D \frac{\partial n}{\partial \rho}\left\langle(\nabla \rho)^{2}\right\rangle+n v\langle|\nabla \rho|\rangle$

$\mathrm{v}(\Phi)=-\mathrm{v} 0\left\langle(\nabla \rho)^{2}\right\rangle /\langle|\nabla \rho|\rangle$ for transport solvers with the definition of flux,

$\Gamma=-D \frac{\partial n}{\partial \rho}\left\langle(\nabla \rho)^{2}\right\rangle-n v\langle|\nabla \rho|\rangle$

- Boundary condition:

\begin{tabular}{|l|l|l|l|}
\hline Time $(\mathrm{s})$ & 100 & 110 & $130-550$ \\
\hline$n_{\text {ea }}\left(10^{19} / \mathrm{m}^{3}\right)$ & 1.8 & 3.0 & 4.6 \\
\hline
\end{tabular}

- Impurity contents:

$\mathrm{n}_{\mathrm{Be}} / \mathrm{n}_{\mathrm{e}}=0.02, \mathrm{n}_{\mathrm{Ar}} / \mathrm{n}_{\mathrm{e}}=0.0005$

Ramp-down phase: $t=550-710$ s $(H$-mode 550-630 s. L-mode 630-710 s)

- Edge particle source for electrons:

$\mathrm{S}(\Phi)=\mathrm{S}_{0} \exp \left[15\left(\Phi-\Phi_{\mathrm{a}}\right) / \Phi_{\mathrm{a}}\right], \mathrm{S}_{0}=7.5 \times 10^{20}$ atom $/ \mathrm{m}^{3}$

- Electron particle diffusivity:

$\mathrm{D}_{\mathrm{e}}(\Phi)=\mathrm{D}_{0}+\mathrm{D}_{1}\left(\Phi / \Phi_{\mathrm{a}}\right)^{2}$ for $\Phi<\Phi_{\text {ped }}$

$\mathrm{D}_{\mathrm{e}}(\Phi)=\mathrm{D}_{2}$ for $\Phi \geq \Phi_{\text {ped }}$

, where $\mathrm{D}_{0}=0.5 \mathrm{~m}^{2} / \mathrm{s}, \mathrm{D}_{1}=1.0 \mathrm{~m}^{2} / \mathrm{s}, \mathrm{D}_{2}=0.1 \mathrm{~m}^{2} / \mathrm{s}$ for $550-630 \mathrm{~s}, \mathrm{D}_{2}=1.27 \mathrm{~m}^{2} / \mathrm{s}$ for $630-710 \mathrm{~s}$, and $\Phi_{\text {ped }}=0.88 \Phi_{\mathrm{a}}$

- Electron pinch number:

$\mathrm{R}_{0} \times \mathrm{V}_{0} / \mathrm{D}=\mathrm{V}_{0} \times\left(\Phi / \Phi_{\mathrm{a}}\right)^{1 / 2}$, where $\mathrm{R}_{0}=6.2 \mathrm{~m}, \mathrm{~V}_{0}=1.385$ and positive sign corresponds the inward pinch.

$\mathrm{v}(\Phi)=\mathrm{v}_{0}$ for transport solvers with the definition of flux, 
$\Gamma=-D \frac{\partial n}{\partial \rho}\left\langle(\nabla \rho)^{2}\right\rangle+n v\left\langle(\nabla \rho)^{2}\right\rangle$

$\mathrm{v}(\Phi)=-\mathrm{v}_{0}$ for transport solvers with the definition of flux,

$\Gamma=-D \frac{\partial n}{\partial \rho}\left\langle(\nabla \rho)^{2}\right\rangle-n v\left\langle(\nabla \rho)^{2}\right\rangle$

$\mathrm{v}(\Phi)=\mathrm{v}_{0}\left\langle(\nabla \rho)^{2}\right\rangle /\langle|\nabla \rho|\rangle$ for transport solvers with the definition of flux,

$\Gamma=-D \frac{\partial n}{\partial \rho}\left\langle(\nabla \rho)^{2}\right\rangle+n v\langle|\nabla \rho|\rangle$

$\mathrm{v}(\Phi)=-\mathrm{v} 0\left\langle(\nabla \rho)^{2}\right\rangle /\langle|\nabla \rho|\rangle$ for transport solvers with the definition of flux,

$\Gamma=-D \frac{\partial n}{\partial \rho}\left\langle(\nabla \rho)^{2}\right\rangle-n v\langle|\nabla \rho|\rangle$

- Boundary condition:

\begin{tabular}{|l|l|l|l|l|l|l|l|l|l|}
\hline Time $(\mathrm{s})$ & 550 & 570 & 590 & 610 & 630 & 650 & 670 & 690 & 710 \\
\hline nea & 4.6 & 4.1 & 3.7 & 3.2 & 2.8 & 0.863 & 0.575 & 0.345 & 0.230 \\
$\left(10^{19} / \mathrm{m}^{3}\right)$ & & & & & & & & & \\
\hline
\end{tabular}

- Impurity contents: $\mathrm{n}_{\mathrm{Be}} / \mathrm{n}_{\mathrm{e}}=0.02, \mathrm{n}_{\mathrm{Ar}} / \mathrm{n}_{\mathrm{e}}=0.0005$ 